\title{
Mechanics of longitudinal and flexural locally resonant elastic metamaterials using a structural power flow approach
}

\author{
Hasan B. Al Ba'ba'a, Mostafa Nouh* \\ Mechanical and Aerospace Engineering Department, University at Buffalo (SUNY), Buffalo, NY 14260, USA
}

\section{A R T I C L E I N F O}

\section{Keywords:}

Elastic metamaterials

Power flow

Stop bands

\begin{abstract}
A B S T R A C T
Elastic metamaterials are sub-wavelength structures with locally resonant components that contribute to the rise of tunable stop bands, i.e. frequency ranges within which waves do not propagate. A new approach is presented here to model and quantify this stop band behavior by evaluating structural vibrating power flowing in the different constituents of locally resonant metamaterials. It is shown that the patterns of power propagation resemble, to a great extent, steady-state wave profiles derived from displacement fields, and can thus be used to develop an algorithm that numerically predicts stop band frequencies for any given realization with a finite length and a known number of repeating cells. The approach is presented here in the context of one-dimensional metamaterials with single and multiple internal resonators and is applied to two traditional examples constituting both longitudinal and flexural type structures. The presence of dissipative elements is taken into consideration since the active component of vibrational power is shown to depend on the damping matrix of the finite element description. The presented approach can be further extended to complex metamaterials with multi-dimensional locally resonant configurations to locate critical energy transmission paths within the media of such structures.
\end{abstract}

\section{Introduction}

Elastic metamaterials are optimally engineered materials which comprise locally housed resonating substructures which contribute to the rise of unique wave dispersive behavior [1-6]. As such, they have recently gained considerable attention for a broad range of applications that require mitigation of vibro-acoustic excitations. The structural dynamics of elastic metamaterials have been investigated in onedimensional bars and rods [7-9] as well as flexural beams [10-19]. The discussion has also been lately extended to two-dimensional membranes and plates [20-23]. Unlike phononic crystals, metamaterials are known for their ability to realize sub-wavelength stop bands which are dependent on the resonant properties of their constituent elements rather than the cell size [24]. As a result, their wave attenuation and directional filtering characteristics can be also tuned using piezoelectrics and active control mechanisms [25-28].

Wave propagation models are typically used to depict the dispersive behavior and theoretically predict stop bands in periodic locally resonant structures. Among these models, Bloch-wave, Floquet models, and the Transfer Matrix Method (TMM) are some of the most wellknown and widely used methods [29-32]. Although stop band behavior exhibited by locally resonant sources does not require periodicity, the existence of a periodic placement allows the analysis of wave propagation and dynamic characteristics on a unit cell basis, thus facilitating the analysis using these methods. Predictions derived from the periodic symmetry of the metamaterial typically exercise a number of assumptions such as infinite length and the lack of end boundary conditions. Consequently, the response of physically realized metamaterials often deviate from the predictions of unit cell dispersion curves. Here, we present a structural power flow model that captures and quantifies stop band behavior in finite metamaterials independent of the presence of a self-repeating unit cell. Furthermore, the proposed technique allows us to enforce boundary conditions which will typically be exhibited in actual designs of elastic metamaterials such as end-cantilevers and/or simple supports.

The structural power flow model, often referred to as a structural intensity analysis (SIA), provides a mathematical tool that can be used to quantify energy flow and transmission paths in vibrating structures [33-39]. The analysis can be integrated with the structural dynamics of any given system to predict the streamlines of power flow within the structure in a manner that is analogous to fluid flow. While a very powerful tool, structural intensity analysis has not yet been applied within the context of metamaterials. In this paper, we investigate the use of the power flow model coupled with a finite element description

\footnotetext{
* Corresponding author.

E-mail address: mnouh@buffalo.edu (M. Nouh).
} 
to study the dynamics of one-dimensional locally resonant metamaterials. In addition to its ability to capture stop band behavior, the proposed model has the added advantage of incorporating different types of structural damping such as, but not limited to, complex frequency dependent elastic moduli of viscoelastic constituents. As will be shown, the active power flow in vibrating structures primarily depends on the damping matrix of the bulk structure thus enabling an accurate modeling tool for dissipative elastic metamaterials. To facilitate the discussion, the SIA analysis is presented here in the context of two benchmark examples from literature $[7,15]$ which are also used to compare the power flow model predictions with traditional wave propagation models. The first example represents a longitudinal bar with sub-masses attached in its cavity that are invisible to an outside observer, while the second is an Euler-Bernoulli beam with dual local resonators.

\section{Structural intensity analysis}

The structural intensity analysis (SIA) is an energy based method used to track power flow and energy paths for purposes of controlling vibrating structures. Here, the analysis is exercised to demonstrate its applicability as an alternative approach to model stop band structures. For a steady-state harmonic analysis, the complex transmitting power is derived from the structure's velocity $\dot{u}$ and exciting force $F$ as follows

$S=\frac{1}{2}\{F\}^{T}\{\dot{u}\}=\frac{1}{2} j \omega\{F\}^{T}\{u\}$

where $\{F\}^{T}$ is the Hermitian transpose of the force vector and the displacement vector $\{u\}$ is calculated from the structural dynamics of the metamaterial

$\{u\}=\left[K_{d}\right]^{-1}\{F\}$

and the dynamic stiffness matrix $\left[K_{d}\right]$ is given by:

$\left[K_{d}\right]=[K]+j \omega[C]-\omega^{2}[M]$

where $[K],[C],[M]$, and $\omega$ are the stiffness matrix, damping matrix, mass matrix, and the angular excitation frequency, respectively. The transmitting power consists of two components: active power $P$ denoting the real part of $S$ and reactive power $Q$ captured by the imaginary part. Hence, we can rewrite the complex vibrating power $(S)$ as:

$S=P+j Q$

By substituting (2) in (1), expressions for $P$ and $Q$ can be formulated as

$P=\frac{1}{2}\{\dot{u}\}^{T}[C]\{\dot{u}\}=\frac{1}{2} \omega^{2}\{u\}^{T}[C]\{u\}$

$Q=\frac{1}{2} \omega\left[\{u\}^{T}[K]\{u\}-\{\dot{u}\}^{T}[M]\{\dot{u}\}\right]$

Incorporating the SIA within a finite element description, the nodal active and reactive power values at discrete locations along the length of spatially continuous structures can be calculated from

$S_{i}=\frac{1}{2} \omega j\left\{F_{i}\right\}^{T}\left\{u_{i}\right\}$

where $\left\{F_{i}\right\}^{T}$ and $\left\{u_{i}\right\}$ are the $i^{\text {th }}$ element force and displacement vectors. For Euler-Bernoulli beams, for instance, the structural power at the $i^{\text {th }}$ element can be expressed as a function of the transverse deflection and angle, $w_{i}$ and $\theta_{i}$, the shear $V_{i}$ and the bending moment $M_{i}$

$S_{i}=\frac{1}{2} \omega j\left[V_{i} w_{i}+M_{i} \theta_{i}\right]$

For systems with a spring connecting two discrete points, A and B, the power transferred from A to B is calculated using [40]

$S_{A}=\frac{1}{2} \omega j\left\{F_{r A}\right\}^{T}\left\{u_{A}\right\}$

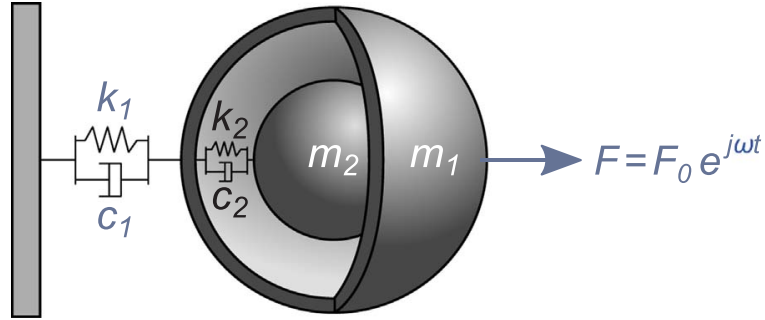

Fig. 1. A 2 DOF mass-in-mass system.

in which $\left\{F_{r A}\right\}^{T}$ is the spring force. Similarly, the net power flowing between A and B may be determined by using

$S_{A B}=\frac{1}{2} \omega j\left\{F_{r A}\right\}^{T}\left\{u_{A}-u_{B}\right\}$

To illustrate, we start with a 2-DOF mass-in-mass system as shown in Fig. 1, with $m_{2} / m_{1}=0.5, c_{2} / c_{1}=0.1$ and $k_{2} / k_{1}=2$. From Eq. (1), the complex vibrating power $(S)$ can be calculated using the global force and the nodal displacement vectors. Alternatively, the active and reactive power can be also found through implementing Eqs. (5) and (6) directly. For $m_{1}=2 \mathrm{~kg}, k_{1}=1 \mathrm{~N} / \mathrm{m}, c_{1}=2 \zeta \sqrt{m_{1} k_{1}}$ and $F_{0}=1 \mathrm{~N}$, Fig. 2 shows the frequency response of both $P$ and $Q$ for damping ratios of $\zeta=0$ and 0.05 . The frequency ratio $\Omega$ is given by $\Omega=\omega / \sqrt{k_{2} / m_{2}}$. Seeing that the active power is a function of the damping matrix, as inferred from Eq. (5), it is expected to have an active power value of zero for the undamped case. In other words, the calculations of active vibrating power in vibrating structures are meaningful only in the presence of damping $[36,37]$. When $\zeta=0.05$, the peaks of $(P)$ reflect the damped natural frequencies of the system. The reactive power $Q$ vanishes at the natural frequencies of the system. However, a zero $Q$ does not necessitate the presence of a system resonance, as seen in Fig. 2 b. Having a damping component is not necessary to calculate the reactive power due to the fact that it depends solely on the difference between the kinetic and potential energies of the system.

\section{Metamaterial bar with local resonators}

To apply a power flow analysis in the context of elastic metamaterials, we first consider a metamaterial bar previously presented by Pai [7]. The mechanism of wave absorption is described here briefly, but we refer the reader to the original paper for more details. The metamaterial consists of a hollow cylindrical bar with a length $\widetilde{L}$, housing spring-mass systems that serve as local resonance sources with a mass, stiffness and damping coefficient of $m, k$, and $c$, respectively, as shown in Fig. 3a. To capture metamaterial dynamics, the analysis discretizes the continuum model into lumped cells at the interface with the resonators as shown in Fig. 3b, c. The bar is supported from one end with a spring of a stiffness $k_{o}$ and excited with a harmonic force $F=F_{0} e^{j \omega t}$, where $F_{o}$ is the sinusoidal force amplitude. The mechanical and geometrical properties of the metamaterial bar are shown in Table 1.

\subsection{Governing equations and dispersion relations}

For the unit cell shown in Fig. $3 \mathrm{~b}$, the equations of motion can be derived as [7]:

$\bar{E} A\left(u_{1}^{\prime}-u_{-1}^{\prime}\right)+k\left(v-u_{o}\right)+c\left(\dot{v}-\dot{u}_{o}\right)=\int_{-L / 2}^{L / 2} \rho A \ddot{u} d x$

$c\left(\dot{u}_{o}-\dot{v}\right)+k\left(u_{o}-v\right)=m \dot{v}$

where $\bar{E}$ is a complex modulus of elasticity which is equal to $E(1+\eta j)$, with $E$ being the storage modulus and $\eta$ representing the material loss factor. Using Bloch's theorem, the deflection of the bar has a solution of $u(x, t)=p e^{j(\beta x-\omega t)}$ where $\beta=2 \pi / \lambda$ is the wavenumber and $\lambda$ is the 

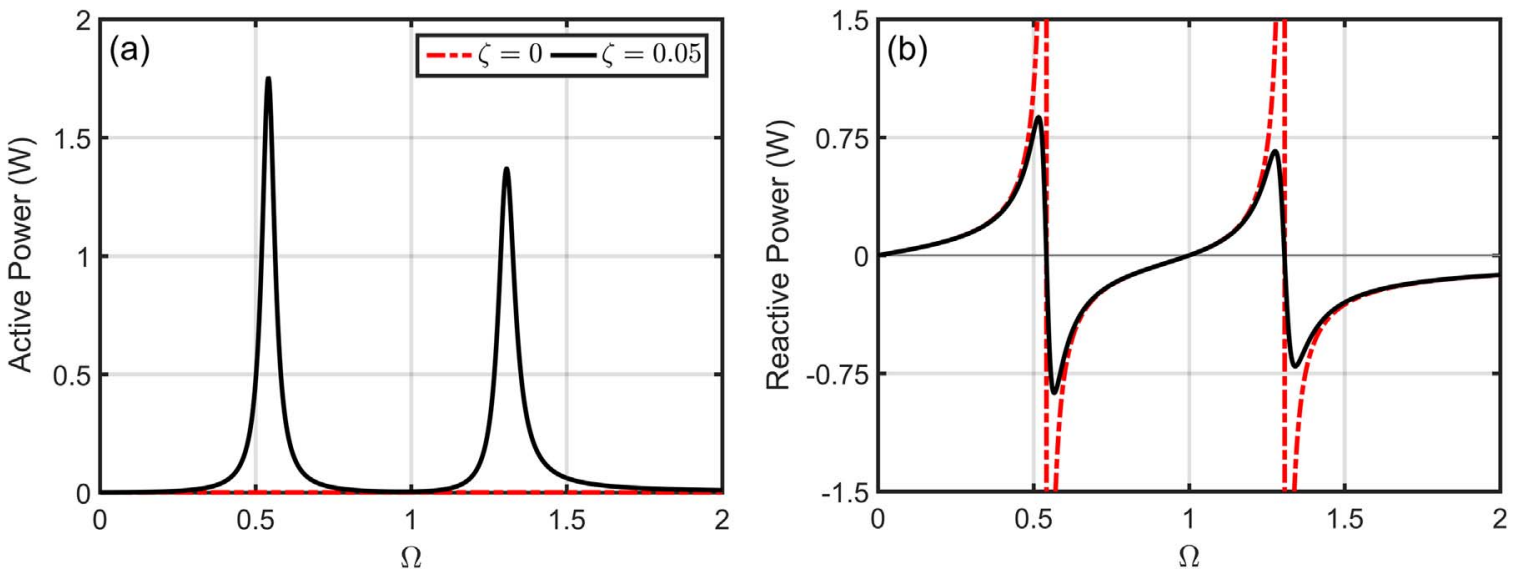

Fig. 2. Frequency response of the total (a) active and (b) reactive vibrational power in a 2-DOF system.

wavelength. The internal masses form a discrete displacement field $v(t)=q e^{-j \omega t}$. Substituting these into the equations of motion yields the dispersion relation

$m \bar{m} \omega^{4}+j c(m+\bar{m}) \omega^{3}-((m+\bar{m}) k+m \bar{k}) \omega^{2}-j c \bar{k} \omega+k \bar{k}=0$

where $\bar{k}=4 \frac{\bar{E} A}{L} \bar{\beta} \sin (\bar{\beta}), \bar{m}=m_{1} \frac{\sin (\bar{\beta})}{\bar{\beta}}$ and $\bar{\beta}=\beta L / 2$. Eq. (13) can be used to compute the band structure of the metamaterials and locate stop band regions. Fig. 4a shows the band diagrams of the metamaterial bar for different values of the internal spring stiffness (represented by the parameter $r$ ). Fig. 4a shows that the spring stiffness affects the shape of the dispersion curve but not the range of the stop band. The stop band behavior can also be determined using the negative effective mass concept $[3,15,7]$, as shown in Fig. $4 \mathbf{b}$ which displays the frequency response of the normalized effective mass $\widetilde{m}=\widetilde{m}_{1} /\left(m+m_{1}\right)$. The stop band spans the range $1<\Omega<\sqrt{1+m / m_{1}}$ which corresponds to negative values of $\widetilde{m}$. The effective mass becomes infinite at $\Omega=1$, when the inertial forces of the vibration absorber acting against the external forces are massive enough to prohibit incident wave propagation. These inertial forces decrease gradually as the frequency approaches the higher bound of the stop band region. Once the exciting frequency passes the stop band region, the internal and external systems become $180^{\circ}$ out of phase yielding the optical vibration mode of the metamaterial.

\subsection{Energy-based stop band prediction}

By evaluating the structural power flow in the metamaterial bar, we introduce a Stop Band Index (SBI) to locate stop bands over a given frequency range. The SBI is a binary indicator of whether a stop band exists (1) or not (0). The algorithm is based on identifying the active structural power value at the unexcited end of the structure while comparing with a minimal numerical threshold. Fig. 5 shows the frequency response function (FRF) of a metamaterial bar (with $r=1$ ) comprised of 400 cells and a uniform one (with the same size and
Table 1

Geometric and physical parameters of the metamaterial bar.

\begin{tabular}{|c|c|c|c|}
\hline Storage Modulus (E) & $70 \mathrm{GPa}$ & Mass Density ( $\rho$ ) & $2800 \mathrm{~kg} / \mathrm{m}^{3}$ \\
\hline Total Length $(\tilde{L})$ & $4 \mathrm{~m}$ & Number of Cells $(n)$ & 400 \\
\hline Cell Length $(L)$ & $0.01 \mathrm{~m}$ & Cross-sectional Area $(A)$ & $75 \pi \times 10^{-6} \mathrm{~m}^{2}$ \\
\hline $\begin{array}{l}\text { Mass ratio } \\
\left(\mathrm{m} / \mathrm{m}_{1}=\mathrm{m} / \rho A L\right)\end{array}$ & 0.3 & Spring stiffness $(k)$ & $m(\omega / r)^{2}$ \\
\hline Damping coefficient $(c)$ & $2 \zeta \sqrt{m k}$ & Damping ratio $(\zeta)$ & $10^{-12}$ \\
\hline Excitation Frequency $(\omega)$ & $2 \pi c_{p} / \lambda$ & Wave speed $\left(c_{p}\right)$ & $\sqrt{E / \rho}$ \\
\hline Force amplitude $\left(F_{o}\right)$ & $k_{o} b_{o}$ & $\begin{array}{l}\text { Base Excitation Amplitude } \\
\left(b_{o}\right)\end{array}$ & $1.0 \mathrm{~mm}$ \\
\hline
\end{tabular}
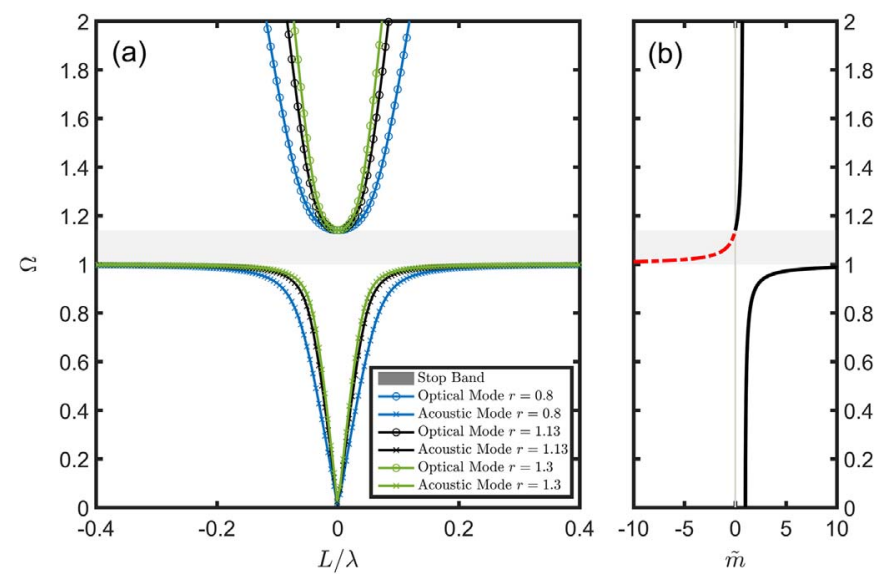

Fig. 4. (a) Dispersion curves for different values of $r$ and (b) the variation of the effective mass of the metamaterial bar with frequency.

properties but without resonators). The response is compared to the SBI showing excellent agreement. This confirms that structural power, similar to elastic waves, is effectively blocked at stop band regions
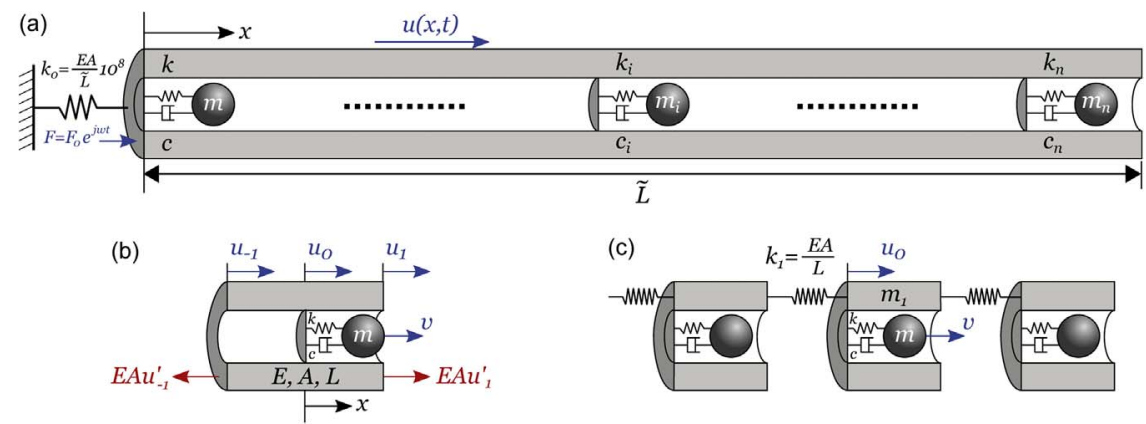

Fig. 3. (a) A locally resonant metamaterial bar, (b) an individual unit cell, and (c) a discretized description of the bar [7]. 


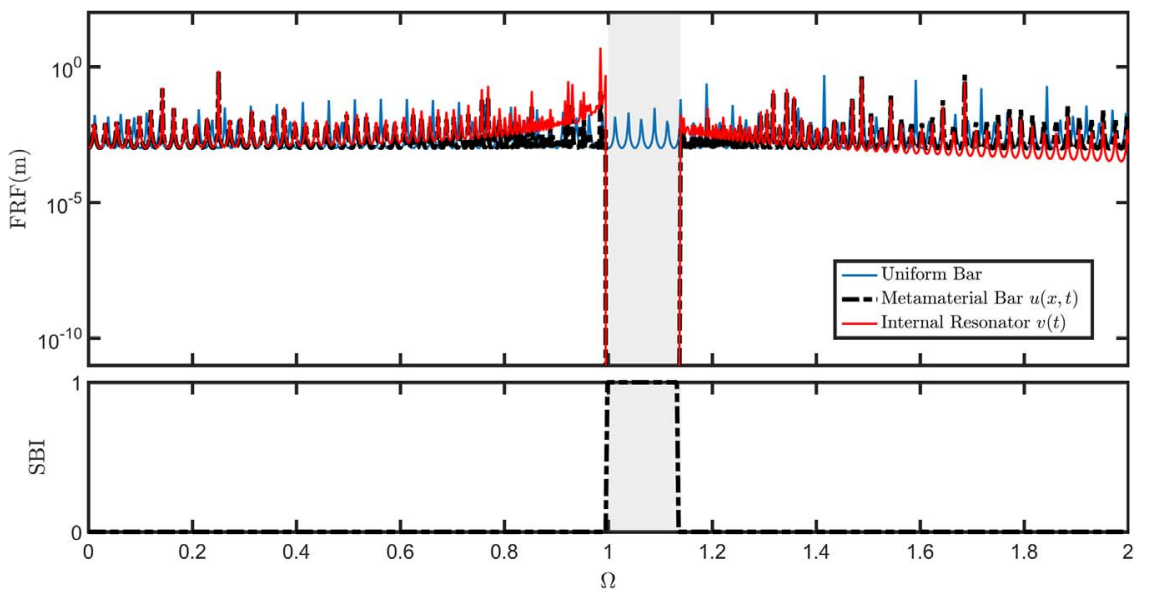

Fig. 5. Comparison between the frequency response function (top) of the metamaterial bar and the developed Stop Band Index (bottom).

and can thus be used as a detecting measure of the same behavior. Since SIA is based on the finite element model of the structure, this approach facilitates the identification of stop bands in metamaterials with complex configurations and under different inputs as no restrictions are placed on the boundary conditions or the excitation locations.

Evaluating the active and reactive power components at different locations along the length of the metamaterial bar gives further insights on structural power at stop band frequencies. Figs. 6 and 7 show the power values at the first and last cells in both the uniform and the metamaterial bar. Outside the stop bands, the active power at the first and last cells, $P_{1}$ and $P_{400}$, in both structures are comparable. The difference between the minimum and maximum values of the active power at pass band regions is not significant. In the stop band region, however, the active power magnitude at the last cell is orders of magnitude smaller than its counterparts at the excited end, a clear indication of power blockage. The difference in the power magnitude at the unexcited end between the uniform and the metamaterial bar is also very large. Both observations suggesting that structural power is barely able to transmit through the system at stop band frequencies. The reactive power plots are more involved. Because of $x$-axis cross-overs at the structure's natural frequencies, it is very challenging to determine whether zero values of $Q$ are caused by natural frequencies or stop bands, making the reactive power an inappropriate measure of the latter. The total power transmitted in the structure, shown in Fig. 8, is calculated by finding the global nodal displacement and force vectors and then applying Eq. (1).

By computing $P$ at the nodal locations, a spatial representation can

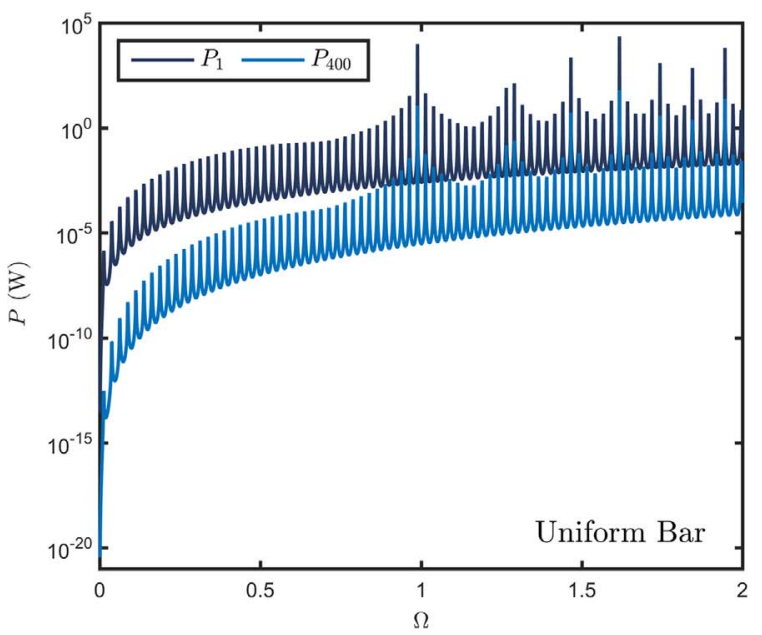

be used to quantify power transmission through the structure along the $x$ - direction, as shown in Fig. 9 for the metamaterial bar, the local resonators and the uniform bar at three excitation frequency ratios $\Omega$ of $0.9,1.1$ and 1.3. The $r$ value used for the spring stiffness is 1 and $\phi$ represents the bar diameter. Using such representation, the blocked power propagation at a stop band frequency (in this case $\Omega=1.1$ ) can be easily observed in both the base structure and the internal resonators of the metamaterial bar (middle column of rows (a) and (b) in Fig. 9).

\subsection{Power flow maps}

Active power flow along the length of one and two-dimensional structures is commonly presented via directional arrows which simultaneously depict the amplitude and direction of structural power flow [41]. In such power flow maps, a series of consecutive arrows emerge from the excitation location and the height of the arrowheads are proportional to the value of $P$ at different $x$ locations. In here, these maps are generated using MATLAB's "quiver" operator. The power flow maps are given for both the uniform and metamaterial bar at the first three vibration modes in Figs. 10 and 11, respectively.

The natural frequencies of the metamaterial bar are slightly lower than the uniform one due to the first being around $30 \%$ heavier. It is important to note here that while the power flow is influenced by the frequency, it differs from the deformation mode shape (plotted for comparison in Figs. 10 and 11). The nodes (zeros) of the mode shapes are not necessarily nodes of the power flow. Since vibrational power flow is a function of the force-velocity product rather than just the

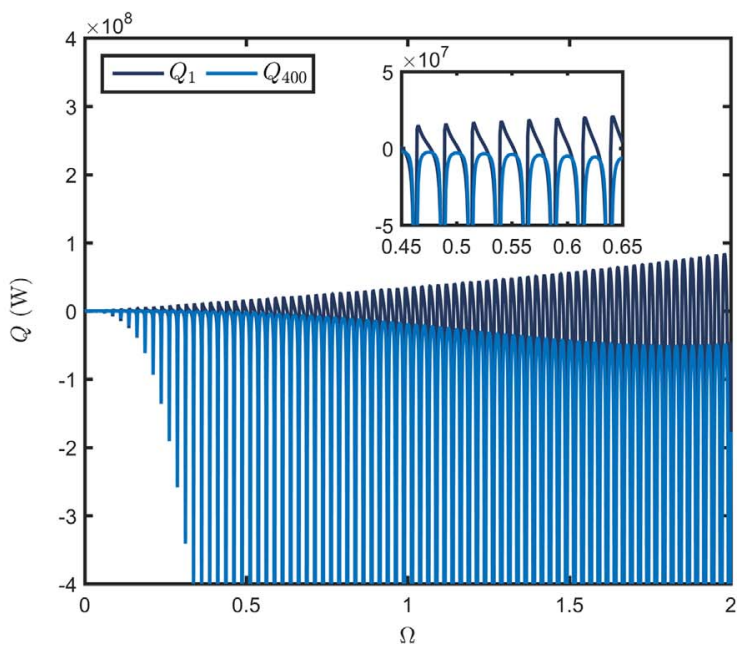

Fig. 6. Active (left) and reactive (right) power components of the uniform bar at the first and the last cells. 

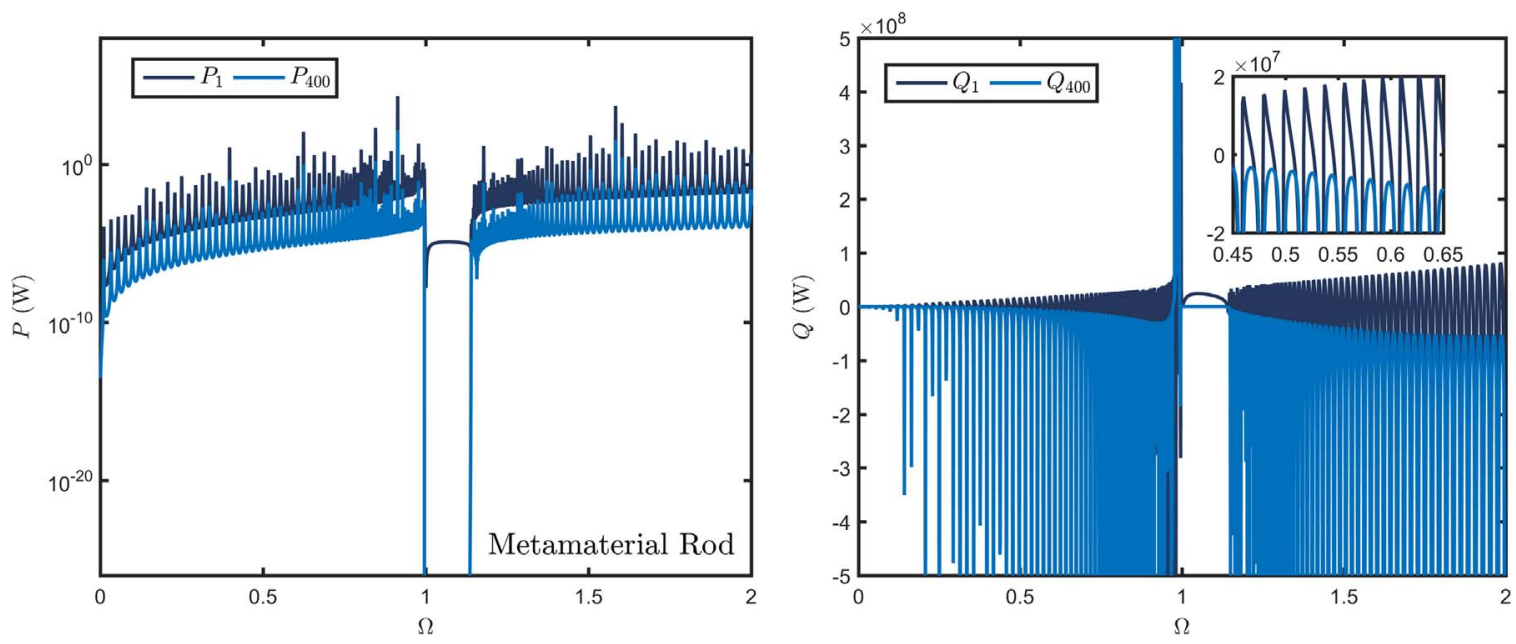

Fig. 7. Active (left) and reactive (right) power components of the metamaterial bar at the first and the last cells.

deflection, $P$ is a maximum at the excitation location and gradually dissipates along the axis of the bar until it reaches a minimum at the free end. It is also important to point out the opposing power flow directions in the base structure and the chain of internal resonators in Fig. 11, a behavior which becomes significant later in the discussion at stop band frequencies.

\subsection{Power flow vs. steady-state displacement field}

Elastic metamaterials are typically explained in the context of steady-state wave profiles which are compared here to the corresponding structural power flow maps. Using a finite element model, the global nodal vector is obtained from a harmonic analysis to plot the wave profiles and subsequently to perform the SIA. For $\lambda=\widetilde{L} / 20$ $(\omega=25 \mathrm{kHz})$ and $m=0.3 \rho A \widetilde{L} / 400$, Fig. 12 shows the displacement field of the metamaterial bar and its resonators ( $1^{\text {st }}$ column), and the corresponding active power flow in both the continuous base structure and the discrete resonators ( $2^{\text {nd }}$ column) corresponding to $r=0.8,1.13$, 1.139, and 1.3.

The wave profiles in Figs. 12a and d show that the motions of the bar and the internal masses are spatially in phase at $r=0.8$, and out of phase at $r=1.3$ representing the acoustic and optic branches of Fig. 4 . Figs. $12 \mathrm{~b}$ and $\mathrm{c}$ show the oscillations at $r=1.13$ and 1.139 which are at the higher bound of the stop band region causing the displacements of both the bar $u(x)$ and the internal masses $v(x)$ to exponentially decay within a number of cells from the excited end. As expected, the wave attenuates faster at the frequency which is further inside the stop band.
The power flow maps given by Fig. 13a show very similar patterns to the displacement profiles. In the two extreme cases $(r=1.3$ and $r=0.8$ ), power flows in the continuous bar from the excitation location (largest magnitude) to the free end. When excited at a stop band frequency $(r=1.13)$, propagation of structural power is prohibited and the quiver arrows quickly vanish within a few number of cells from the excited end. As we move towards the upper bound of the stop band $(r=1.139)$, structural power propagates further along the bar in agreement with the wave profile. Pai attributed the existence of stop bands in the metamaterial bar to a mechanism similar to traditional vibration absorbers [7] spanning a larger frequency range. In Fig. 13b, the power flow in the discrete internal resonator opposes the flow in the base structure as a result of the internal inertial forces counteracting the external excitation force in the metamaterial. An effect which is magnified in the stop band range culminating in a near-perfect power absorption.

\subsection{Metamaterial bar with few local resonators}

The response of the metamaterial bar is investigated with a single internal resonator (at $x=2 \mathrm{~m}$ ). For a wavelength of $\lambda=\widetilde{L} / 8.25$ $(\omega=10.3 \mathrm{kHz})$ and an internal mass of $m=\rho A \widetilde{L} / 40$, Fig. 14 illustrates the wave profiles and the corresponding power flow maps at $r=0.9,1.0$, and 1.1. The single resonator acts as a perfect vibration absorber and, hence, the stop band occurs exactly at the excitation that matches the resonator's natural frequency, whereas considerable attenuation of the elastic wave is still observed in neighboring frequencies. This is in contrast
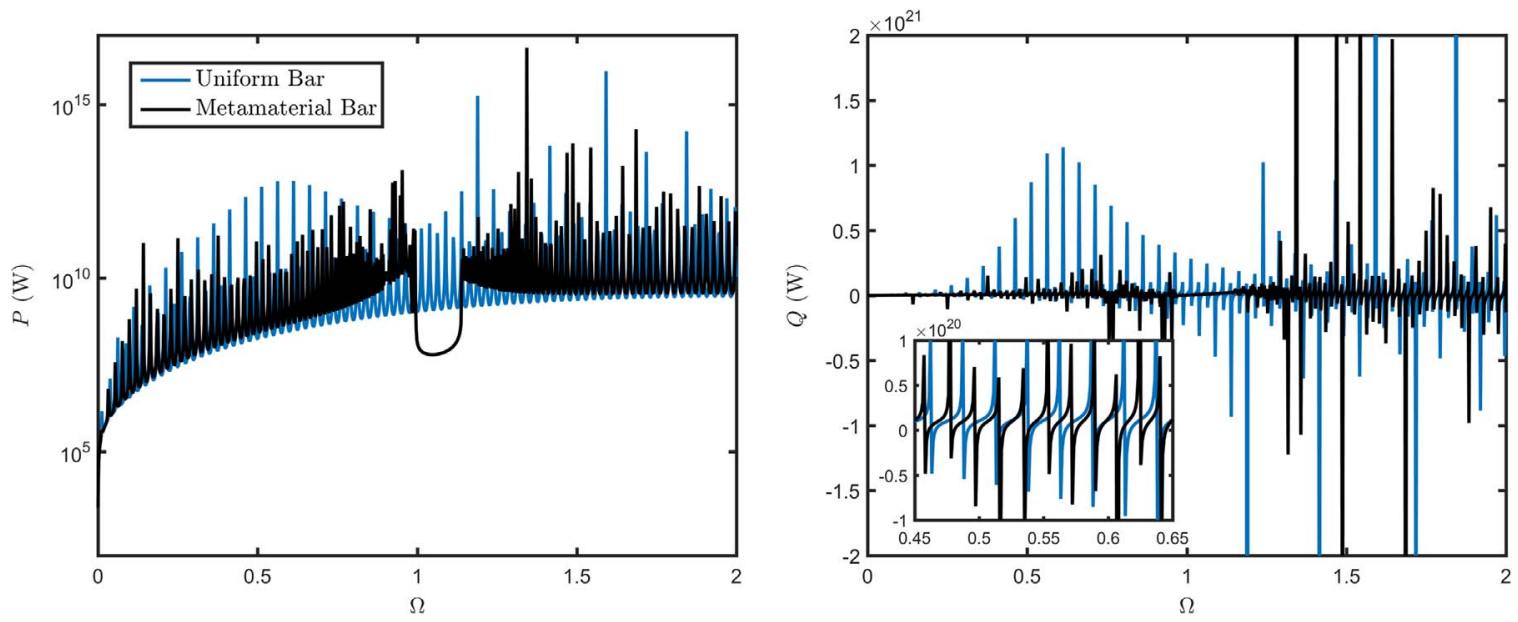

Fig. 8. Total active (left) and reactive (right) power of the uniform and metamaterial bars. 

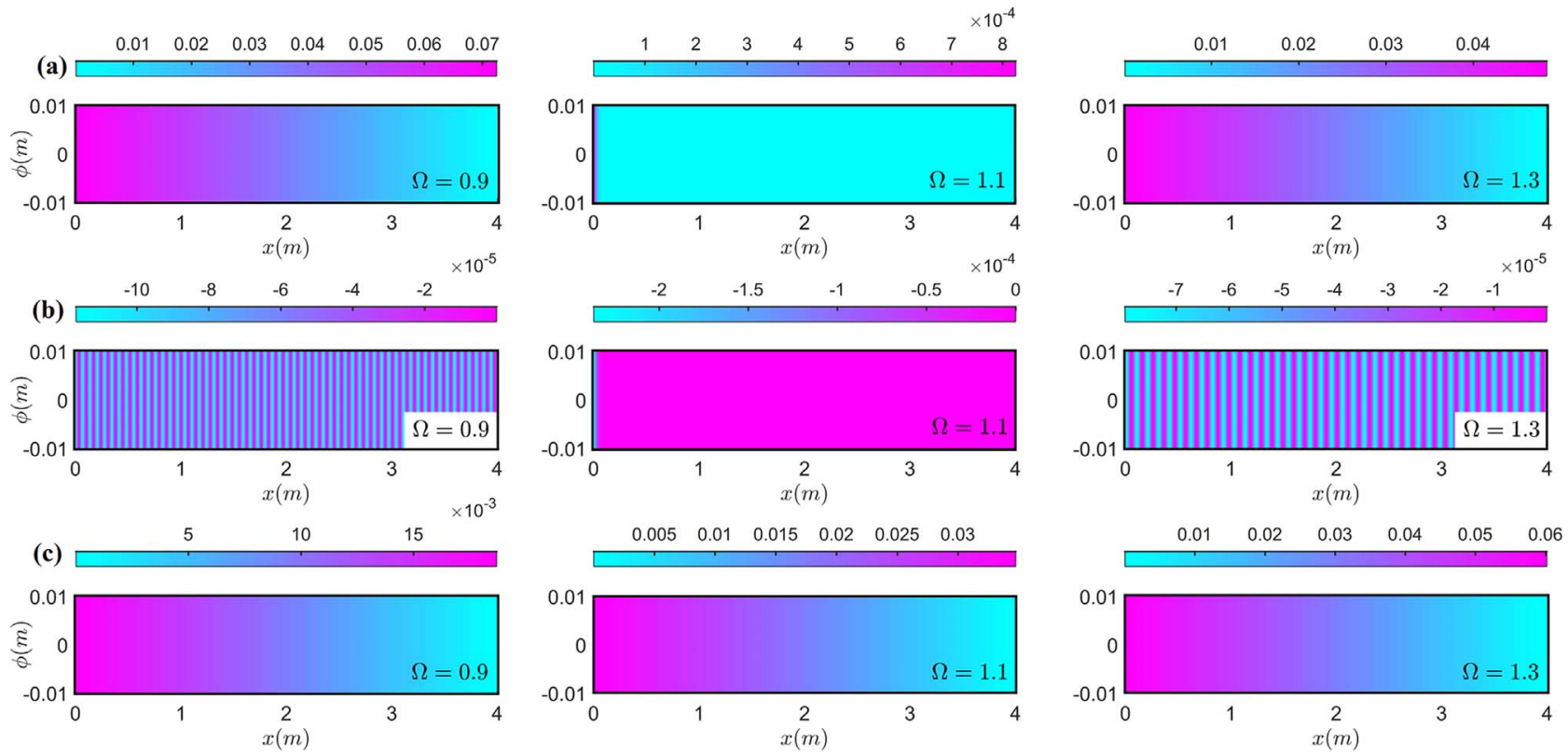

Fig. 9. Variation of vibrational power along the length of (a) the metamaterial bar, (b) the resonators and (c) the uniform bar at $\Omega=0.9,1.1$, and 1.3 .

to the bar's behavior with an array of resonating cells contributing to the rise of an attenuation band over a continuous frequency range. The dynamics of the same metamaterial bar with six resonators that are evenly spaced between $x=2$ and $x=2.4$ are given for the same $r$ values in Fig. 15 . Pai used that example to explain the predominant role of the optic mode in the formation of locally resonant stop bands [7]. The computed power flow maps confirm this hypothesis. In the acoustic mode, it can be clearly seen that active structural power is able to propagate the resonator-filled section of the metamaterial bar but is perfectly blocked at the resonators' natural frequency. In the optic mode, however, flowing power decays each time it passes through an absorber and while perfect power absorption is not realized, a significant reduction in amplitude is thereby achieved after the sixth and last absorber.

\section{Multi-frequency metamaterial euler-bernoulli beam}

Next, the structural intensity analysis is presented in the framework of a metamaterial beam undergoing flexural deformation. Fig. 16a displays the beam and illustrates the associated degrees of freedom for each node in the finite element description [15]. Fig. 16b shows the free body diagram of the discretized unit cell used to derive the governing motion equations. The elastic metamaterial beam is equipped with 100 two-resonator attachments and is simply supported from both its ends.
The beam is excited with a harmonic force of an amplitude of $F_{0}=100 \mathrm{~N}$ at the first cell. For simplicity, we consider an EulerBernoulli beam model (as opposed to the Timoshenko model in the original paper). The different beam parameters are summarized in Table 2.

\subsection{Governing equations and dispersion relations}

The three equations of motion of a unit cell of the metamaterial beam can be derived as [15]

$\int_{-L / 2}^{L / 2} \rho A \ddot{w} d x+\bar{E} I\left(w_{1}^{\prime \prime \prime}-w_{-1}^{\prime \prime \prime}\right)+c_{2}\left(\dot{w}_{0}-\dot{u}_{2}\right)+k_{2}\left(w_{0}-u_{2}\right)=0$

$m_{2} \ddot{u}_{2}+\left(c_{2}+c_{3}\right) \dot{u}_{2}+\left(k_{2}+k_{3}\right) u_{2}-c_{3} \dot{u}_{3}-k_{3} u_{3}-c_{2} \dot{w}_{0}-k_{2} w_{0}=0$

$m_{3} \ddot{u}_{3}+c_{3}\left(\dot{u}_{3}-\dot{u}_{2}\right)+k_{3}\left(u_{3}-u_{2}\right)=0$

Similar to Section 3.1, the dispersion relations are developed by substituting the harmonic wave solution in the equations of motion which, here, yields

$\alpha_{0} \omega^{6}+j \alpha_{1} \omega^{5}-\alpha_{2} \omega^{4}-j \alpha_{3} \omega^{3}+\alpha_{4} \omega^{2}+j \alpha_{5} \omega-\alpha_{6}=0$
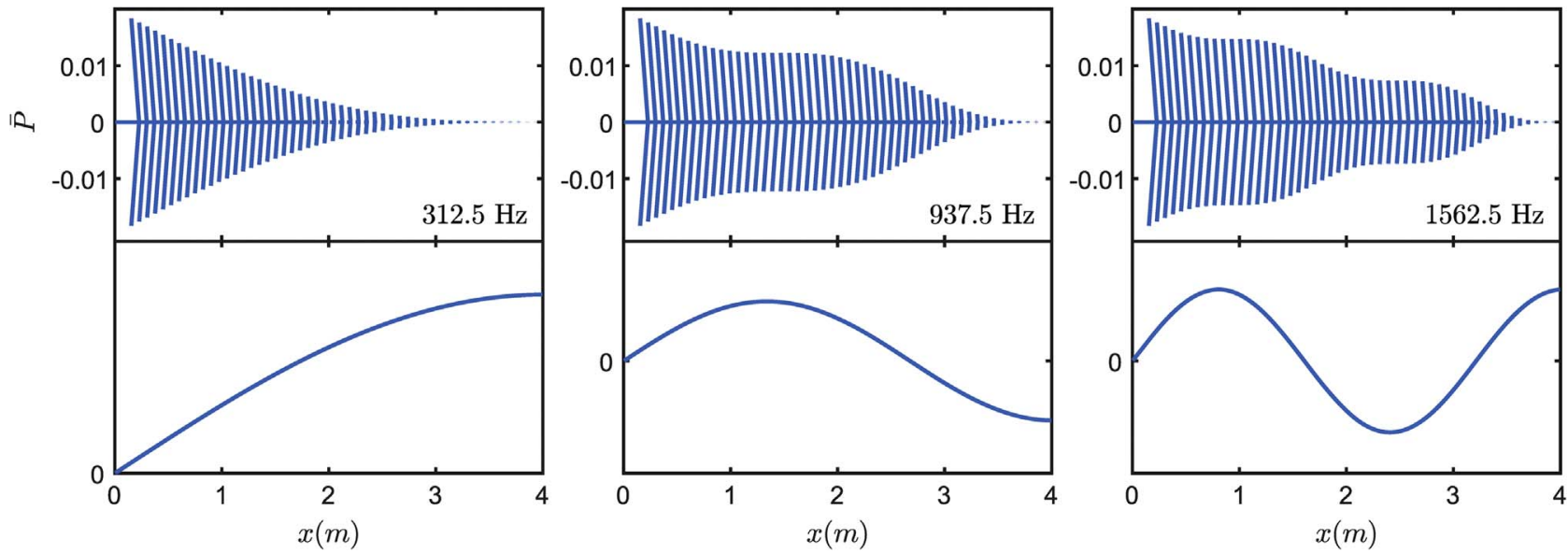

Fig. 10. Power flow maps (top) and the corresponding axial deformation mode shapes (bottom) for the uniform bar at the first three natural frequencies. 

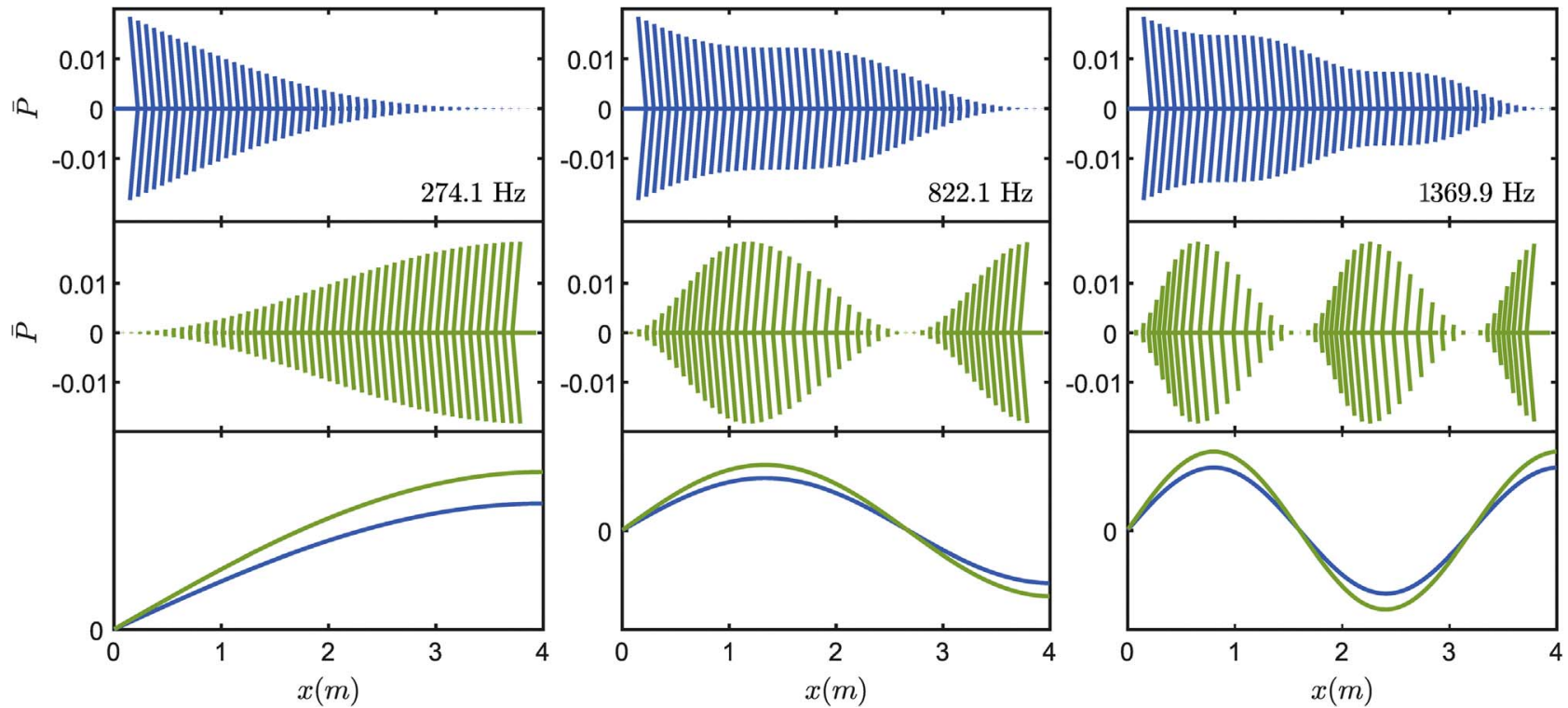

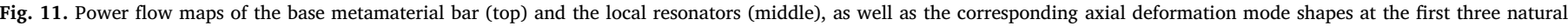
frequencies(bottom).

where

$$
\begin{aligned}
& \alpha_{0}=\bar{m} m_{2} m_{3} \\
& \alpha_{1}=\left(\left(c_{2}+c_{3}\right) m_{3}+c_{3} m_{2}\right) \bar{m}+c_{2} m_{2} m_{3} \\
& \alpha_{2}=\left(\bar{m}+m_{2}+m_{3}\right) c_{2} c_{3}+\left(\left(k_{2}+k_{3}\right) m_{3}+k_{3} m_{2}\right) \bar{m}+\left(\bar{k}+k_{2}\right) m_{2} m_{3} \\
& \alpha_{3}=\left(\bar{m}+m_{2}+m_{3}\right)\left(c_{3} k_{2}+c_{2} k_{3}\right)+\left(c_{2} m_{3}+c_{3}\left(m_{2}+m_{3}\right)\right) \bar{k} \\
& \alpha_{4}=\left(c_{2} c_{3}+k_{2} m_{3}+k_{3}\left(m_{2}+m_{3}\right) \bar{k}+\left(\bar{m}+m_{2}+m_{3}\right) k_{2} k_{3}\right. \\
& \alpha_{5}=\left(c_{2} k_{3}+c_{2} k_{3}\right) \bar{k} \\
& \alpha_{6}=\bar{k} k_{2} k_{3}
\end{aligned}
$$

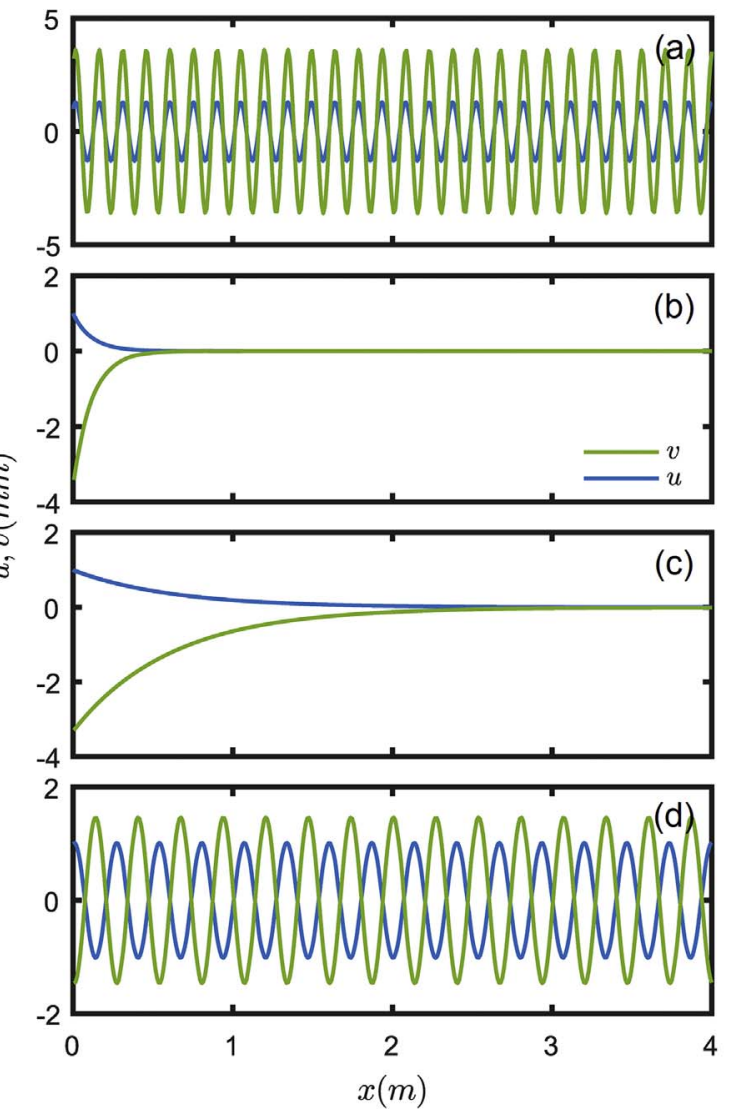

with $\bar{m}=2 \rho A \sin (\beta L / 2) / \beta$ and $\bar{k}=2 \bar{E} I \beta^{3} \sin (\beta L / 2)$. The homogenized stiffness and mass of the beam cell segment are functions of the wavenumber $\beta$, enabling the prediction of stop band frequencies. An additional stop band will emerge here since two different sets of resonators are used. Alternatively, the negative effective mass concept can also be used as an indication of the dominance of internal inertial forces at certain frequencies effectively blocking incident waves. Here, the second stop band (formed as a result of the additional resonator) is tuned to be adjacent to the first stop band by selecting two internal
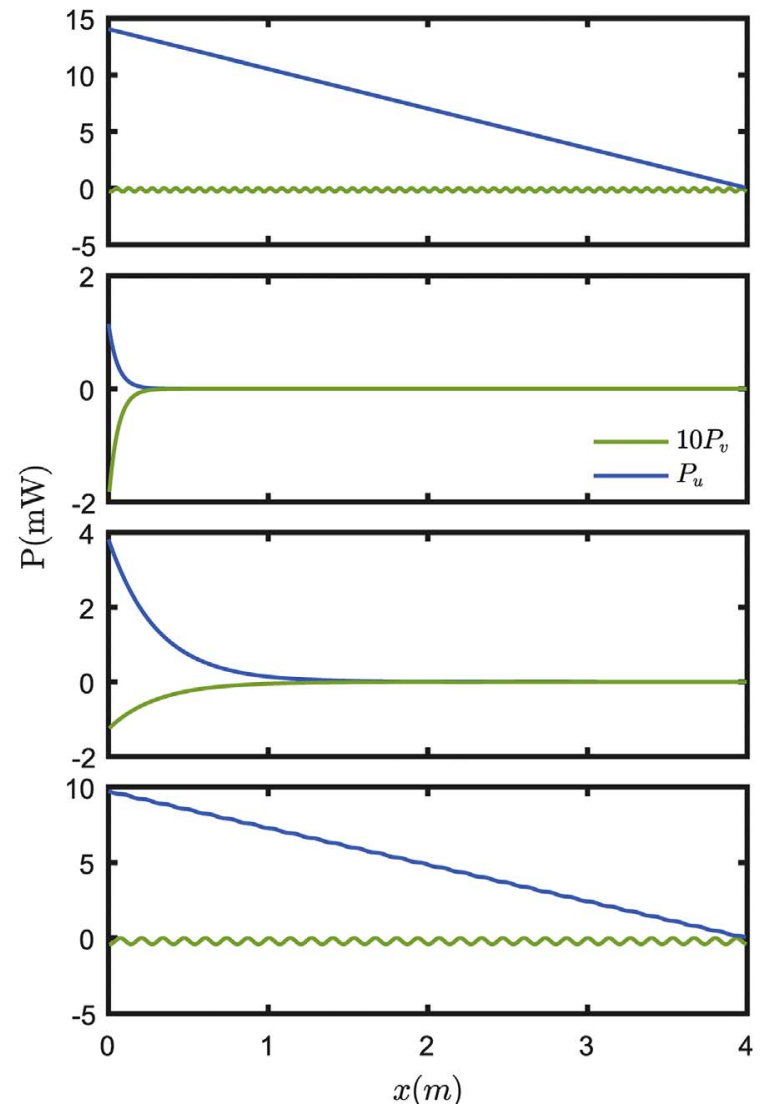

Fig. 12. steady-state wave profiles for the metamaterial bar and the internal masses (left) and the active power (right) for $r=$ (a) 0.8 , (b) 1.13 , (c) 1.139 , and (d) 1.3 . 
(a)

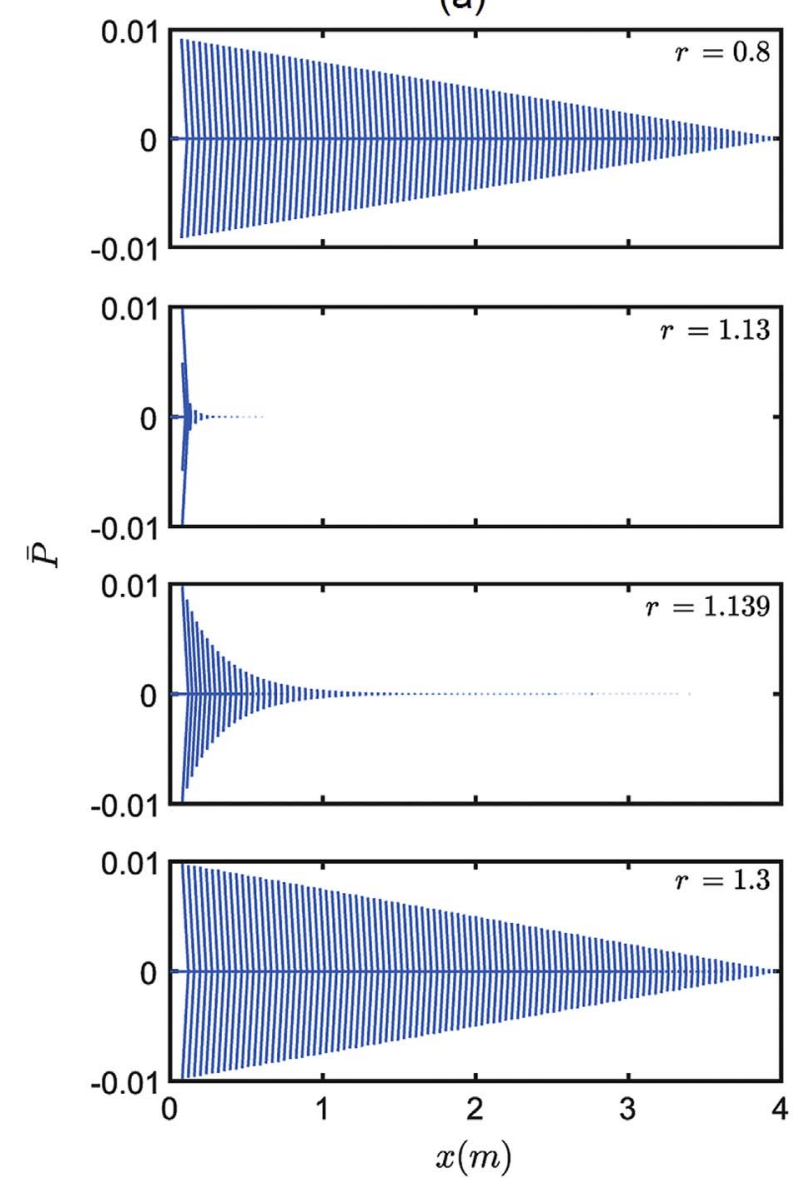

(b)
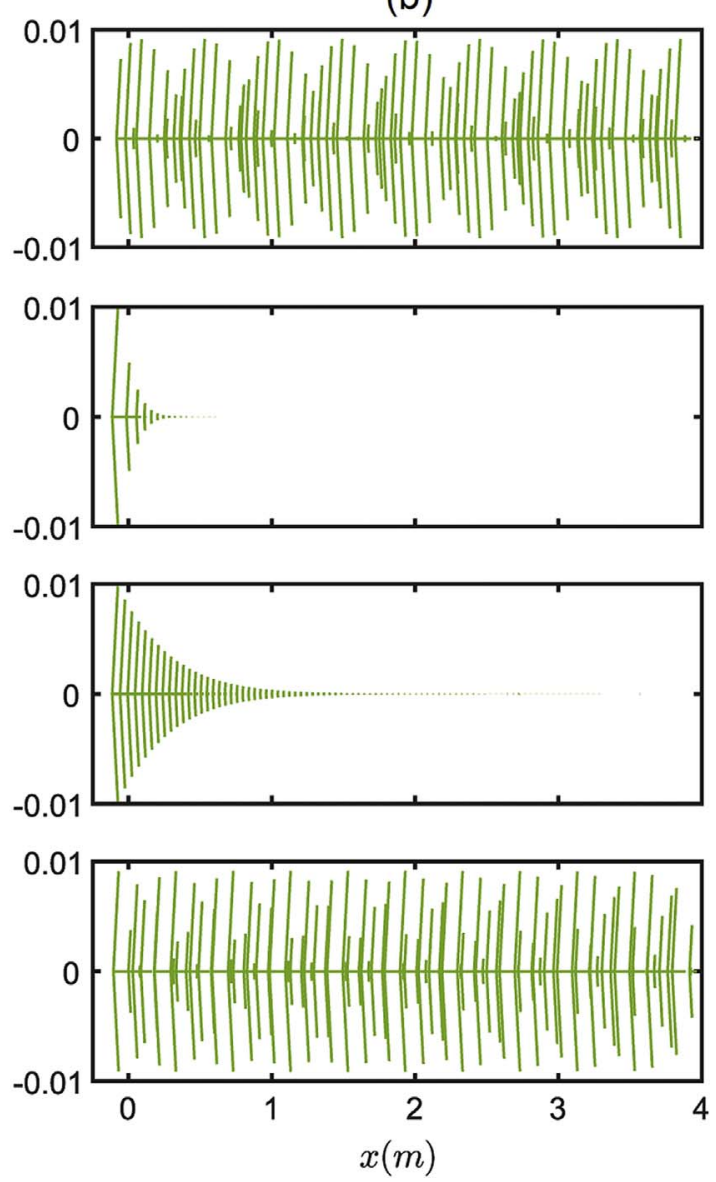

Fig. 13. Power flow maps of (a) the metamaterial bar and (b) the internal masses for $r=0.8,1.13,1.139$, and 1.3.

systems with fairly similar natural frequencies $[4,15]$.

By treating the 3-DOF system shown in Fig. 17a as a single DOF system which comprises internal masses that are not visible to an external observer, a mathematical representation of a dynamic effective mass $\widetilde{m}_{1}$ for the homogenized unit cell can be derived as

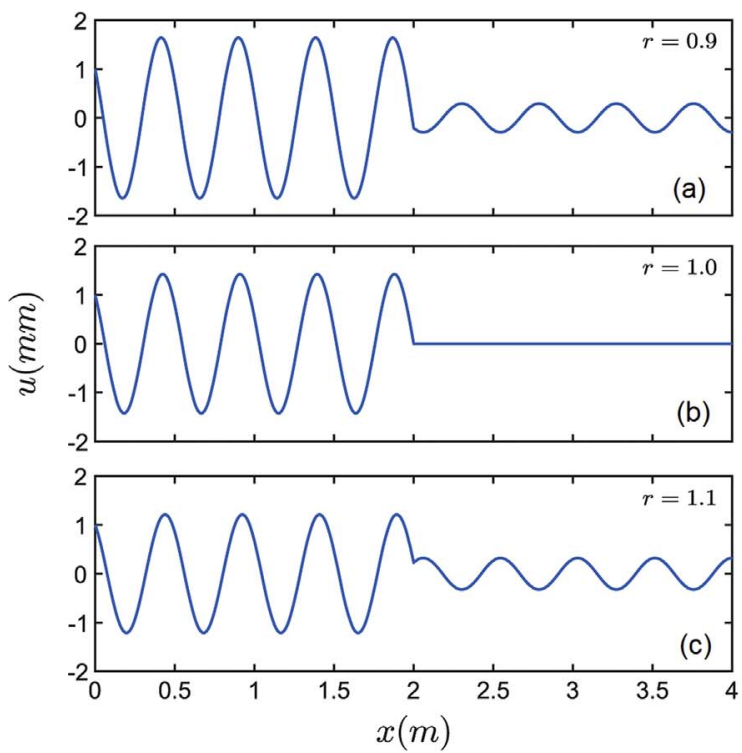

$\widetilde{m}_{1}=m_{1}+m_{2} \frac{(1+\gamma)-\Omega_{3}^{2}}{\Omega_{3}^{2} \Omega^{2}-\left(\Omega_{3}^{2}+(1+\gamma) \Omega^{2}\right)+1}$

where $\Omega_{3}=\frac{\omega}{\omega_{3}}$ and $\gamma=\frac{m_{3}}{m_{2}}$. If the mass ratio equals the spring ratio of the two resonators $\left(\gamma=\frac{m_{3}}{m_{2}}=\frac{k_{3}}{k_{2}}\right)$, in other words both internal systems have the same natural frequency and $\Omega_{3}=\Omega$, the equation simplifies to

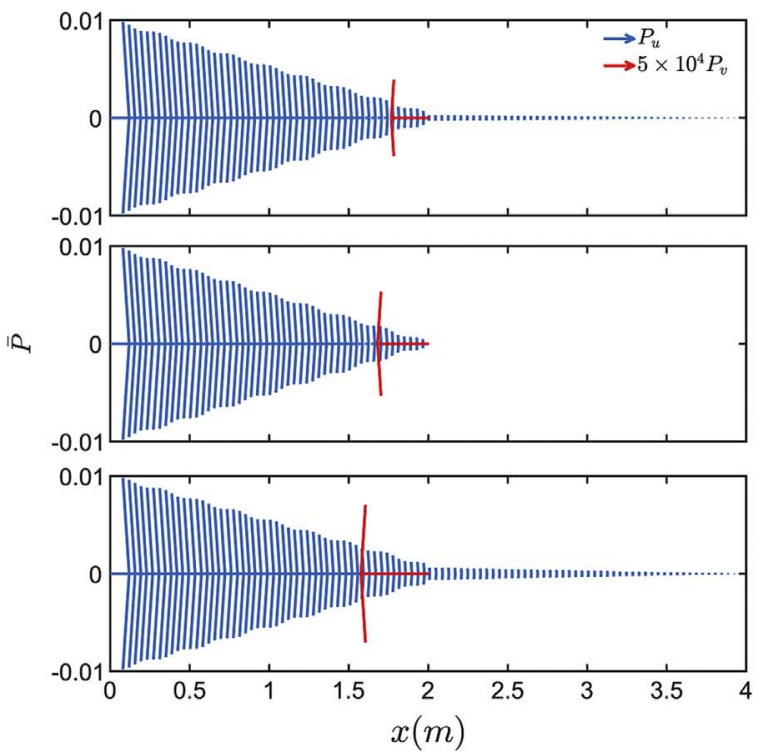

Fig. 14. steady-state wave profiles (left) and the corresponding power flow maps (right) for a metamaterial bar with a single middle resonator for $r=$ (a) 0.9 , (b) 1.0, and (c) 1.1. 

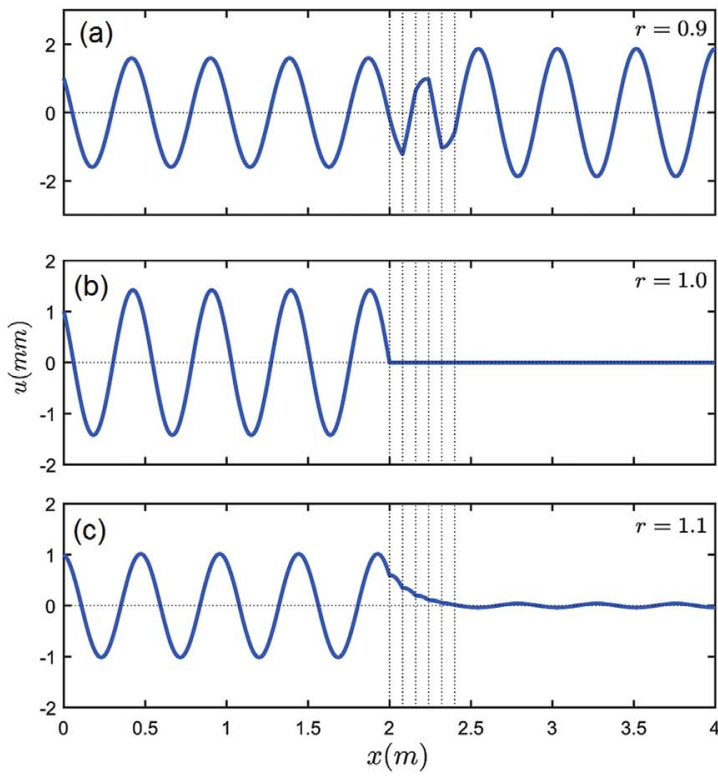
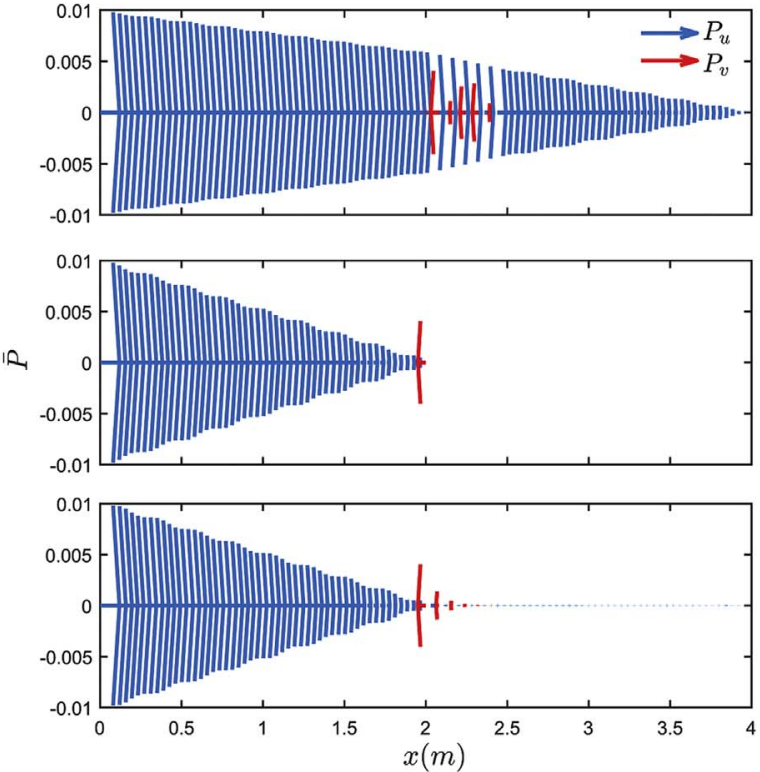

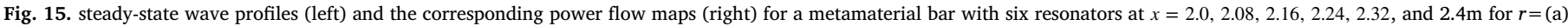
0.9 , (b) 1.0 , and (c) 1.1 .

$\widetilde{m}_{1}=m_{1}+m_{2} \frac{(1+\gamma)-\Omega^{2}}{\Omega^{4}-(2+\gamma) \Omega^{2}+1}$

Without a second internal resonator, $\gamma$ becomes equal to zero and Eq. (19) reduces to the effective mass equation of a 2-DOF mass-in-mass system used in the calculations of the metamaterial bar earlier. For $\gamma=0.05$, Fig. 18 shows the dispersion curves and the normalized effective mass $\left(\widetilde{m}=\widetilde{m}_{1} /\left(m_{1}+m_{2}+m_{3}\right)\right)$ of the unit cell of the metamaterial beam as a function of $\Omega$. The two stop bands span the regions $0.894<\Omega<0.922$ and $1.118<\Omega<1.14$. As expected, the negative effective mass regions match the predicted stop bands from the dispersion relation. The denominator of the second term in Eq. (19) is a function of the mass ratio $\gamma$ and has two repeated roots. A negative $\widetilde{m}_{1}$ is obtained when the second term of the equation becomes negative and its absolute value exceeds $m_{1}$. The acoustic and optic modes in this case correspond to $\Omega<0.894$ and $\Omega>1.14$, respectively. Unlike the single resonator scenario, a mixed mode is observed in the range $0.922<\Omega<1.118$ which is dominated by whichever mode is closer to the exciting frequency.
Table 2

Geometric and physical parameters of the metamaterial beam.

\begin{tabular}{|c|c|c|c|}
\hline Storage Modulus (E) & $72.4 \mathrm{GPa}$ & Mass Density $(\rho)$ & $2780 \mathrm{~kg} / \mathrm{m}^{3}$ \\
\hline Total Length $(\tilde{L})$ & $1 \mathrm{~m}$ & Number of Cells $(n)$ & 100 \\
\hline Cell Length $(L)$ & $0.01 \mathrm{~m}$ & $\begin{array}{l}\text { Cross-sectional Area } \\
\text { (A) }\end{array}$ & $15 \times 10^{-6} \mathrm{~m}^{2}$ \\
\hline Second mass $\left(m_{2}\right)$ & $=0.1 m_{1}=0.1 \rho A L$ & Third mass $\left(m_{3}\right)$ & $=0.05 \mathrm{~m}_{2}$ \\
\hline Spring stiffness $\left(k_{i}\right)$ & $m_{i}\left(\omega_{n}\right)^{2}$ & Local Resonance $\left(\omega_{n}\right)$ & $1000 \mathrm{~Hz}$ \\
\hline $\begin{array}{l}\text { Damping coefficient } \\
\quad\left(c_{i}\right)\end{array}$ & $2 \zeta \sqrt{m_{i} k_{i}}$ & Moment of Inertia $(I)$ & $11 \times 10^{-12} \mathrm{~m}^{4}$ \\
\hline
\end{tabular}

\subsection{Energy-based stop band prediction}

Analogous to the metamaterial bar discussion, a stop band index (SBI) based on power flow in the metamaterial beam is developed to numerically predict the location of stop band frequencies as an alternative tool to the dispersion relations. The SBI prediction is compared with the frequency response of the beam in Fig. 19 showing good agreement. The ability of the SBI to accurately depict the location of the stop bands is confirmed by Fig. 20 which shows drastic drops in

(a)
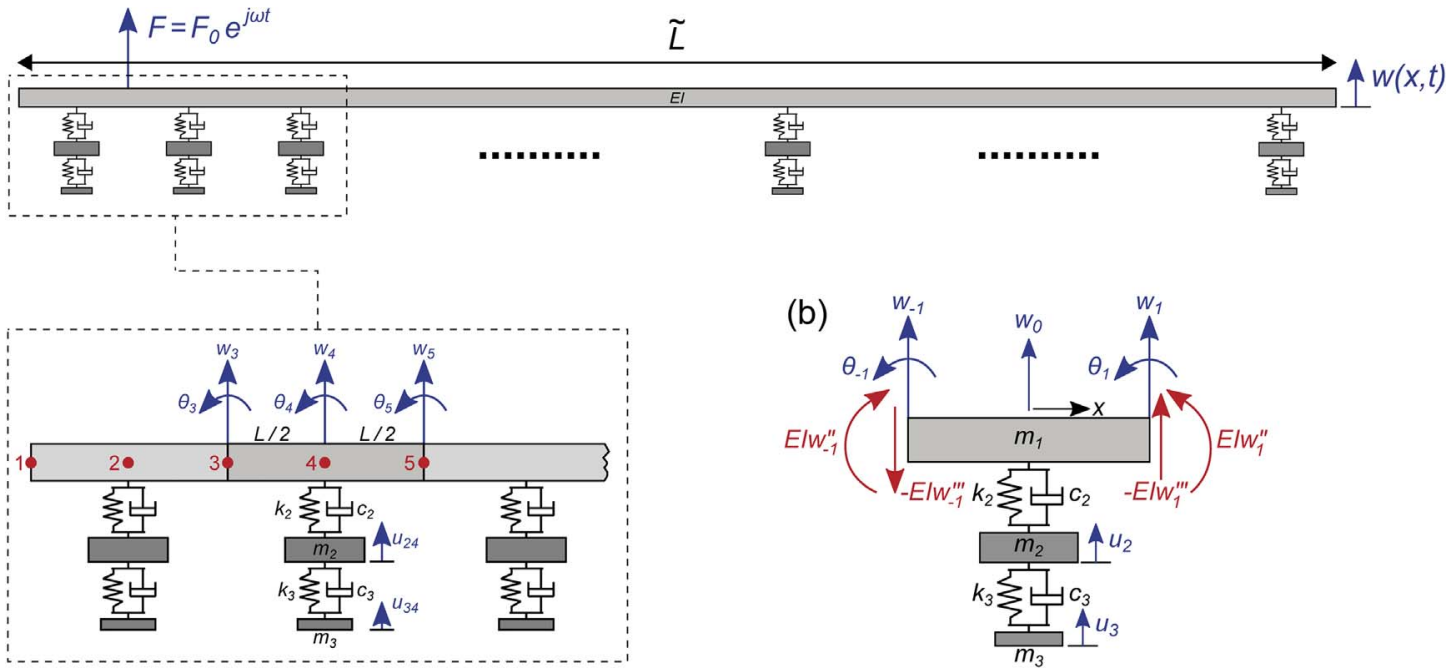

Fig. 16. (a) The metamaterial beam and the associated nodal degrees of freedom in the finite element model and (b) free body diagram of the unit cell [15]. 

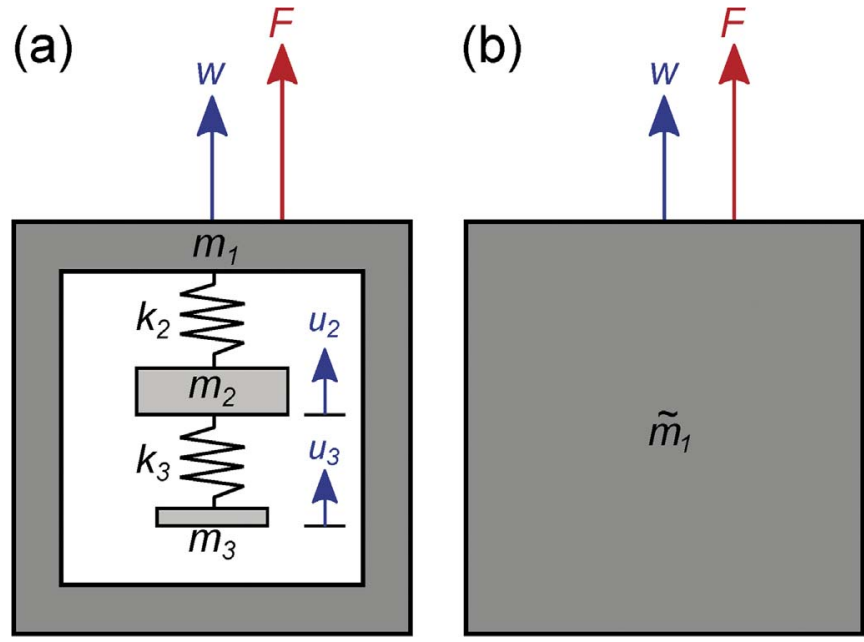

Fig. 17. (a) A 3-DOF discrete system and (b) its effective mass.
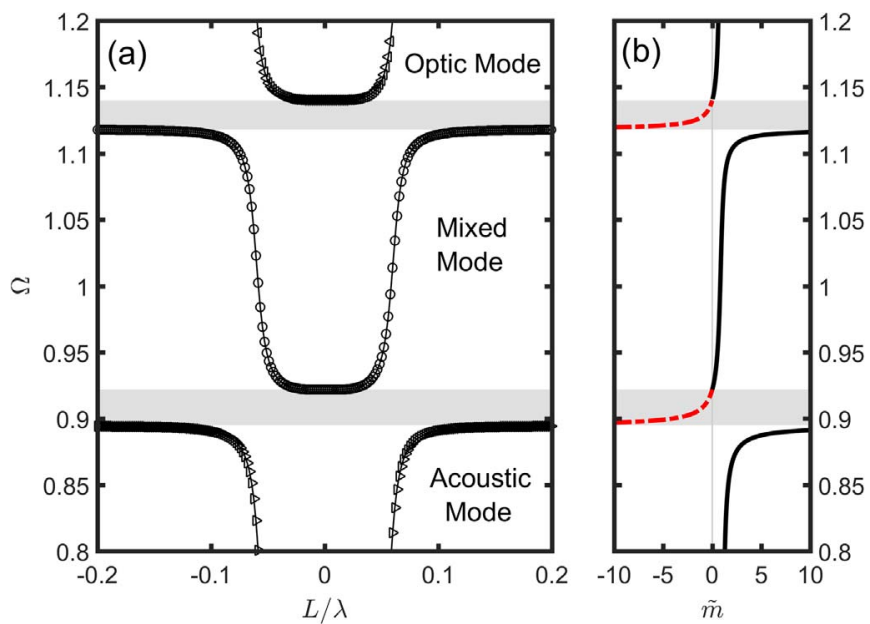

Fig. 18. (a) Dispersion curves and (b) effective mass of the two-resonator metamaterial beam.

the active power propagation at stop band frequencies by comparing the value of $P$ at the beginning and end of the multi-resonator metamaterial beam.

\subsection{Power flow maps}

The power flow maps for the metamaterial beam and a resonator-

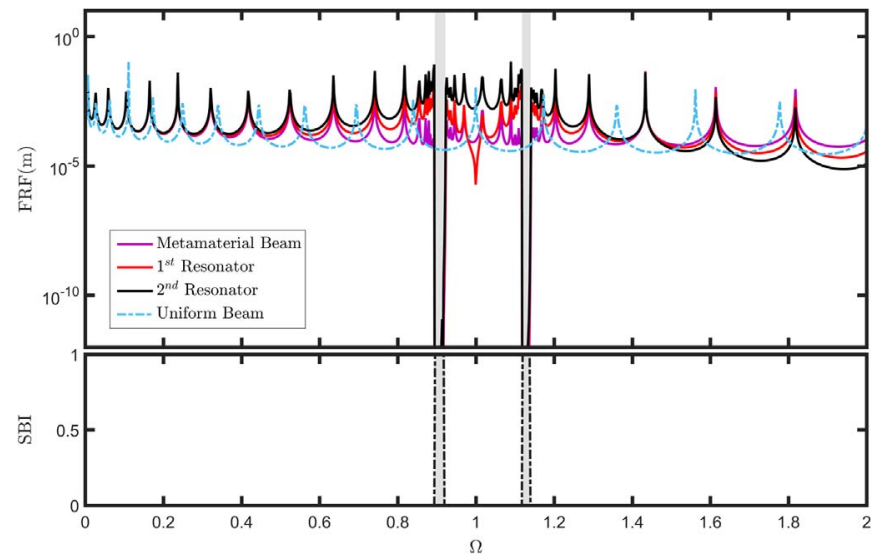

Fig. 19. Comparison between the frequency response function (top) of the metamaterial beam and the developed Stop Band Index (bottom). free uniform beam of the same length are given at the first three bending vibration modes in Figs. 21 and 22, respectively. Since the metamaterial beam is about $10.5 \%$ heavier, its natural frequencies are slightly lower than the uniform beam. Finally, power flow maps are evaluated at the boundaries, and inside, both stop bands of the metamaterial beam. Similar to the metamaterial bar example, the displacement and internal forces vectors are computed from the finite element method to implement the SIA analysis. Fig. 23 shows the bending (transverse) displacement field of the metamaterial beam and the two internal local resonators (1st column), and the variation of active power along the $x$ - direction (2nd column) for $\Omega=0.7,0.9,1$, 1.12, and 1.3.

At $\Omega=0.7$, structural power freely propagates to the unexcited end of the beam which spatially oscillates in phase with both sets of internal masses satisfying the acoustic branch of the dispersion curves. As the excitation frequency increases, the beam goes into its first stop band (at $\Omega=0.9$ ) where the displacement of the beam $w(x)$ along with the two local resonances, $m_{2}$ and $m_{3}$, exponentially decay within the first quarter of the beam's length. The same behavior is repeated at the second stop band (at $\Omega=1.12$ ). In the corresponding power flow maps (Fig. 24), active power in the beam and both resonators dissipates completely at stop band frequencies. The opposing flow direction in the maps of $m_{2}$ and $m_{3}$ is expected since the absorbers work against the external force. It can also be seen in Fig. 24a through e that the inertial forces in the resonators resist the motion in the continuous structure at all the considered frequencies albeit manages to block its propagation only at stop band frequencies. Outside these frequencies, power flows from the excited to the unexcited end where it reaches a minimum value.

\section{Conclusions}

A numerical investigation of vibrational power flow in locally resonant elastic metamaterial has been presented. Integrated with the finite element description, the Structural Intensity Analysis (SIA) has been demonstrated to predict the magnitude and direction of structural power in the different components of the metamaterial. The use of vibrational power flow to predict and quantify stop band frequencies has been presented as an alternative mechanism to the conventional wave dispersion analysis as well as the negative effective mass methods in the context of both longitudinal and flexural metamaterials with single and dual resonators. The power flow maps confirm the hypothesis that the local resonators counteract the external excitations with discrete inertial forces that, within stop band regions, nearly cancel out the power injected in the system and effectively block wave propagation to the unexcited end. The use of this approach also both enables and accurately depicts the performance of dissipative metamaterials and/or metamaterials with dissipative resonators since it is shown that the active power component solely depends on the damping matrix and the dissipative energy within the structure. Furthermore, the proposed approach facilitates the discussion and optimization of actual metamaterial designs since the analysis is directly applied to a physical realization of any metamaterial configuration with a finite length, and a known number of cells, rather than an infinitely long theoretical structure with no end boundary conditions as typically assumed by the traditional wave-based models.

\section{Acknowledgments}

This work was supported by the US National Science Foundation under grant CMMI1647744.

\section{Appendix A. Finite element modeling}

The finite element method is used to calculate the nodal displacements of the metamaterial bar and its local resonators and then perform 

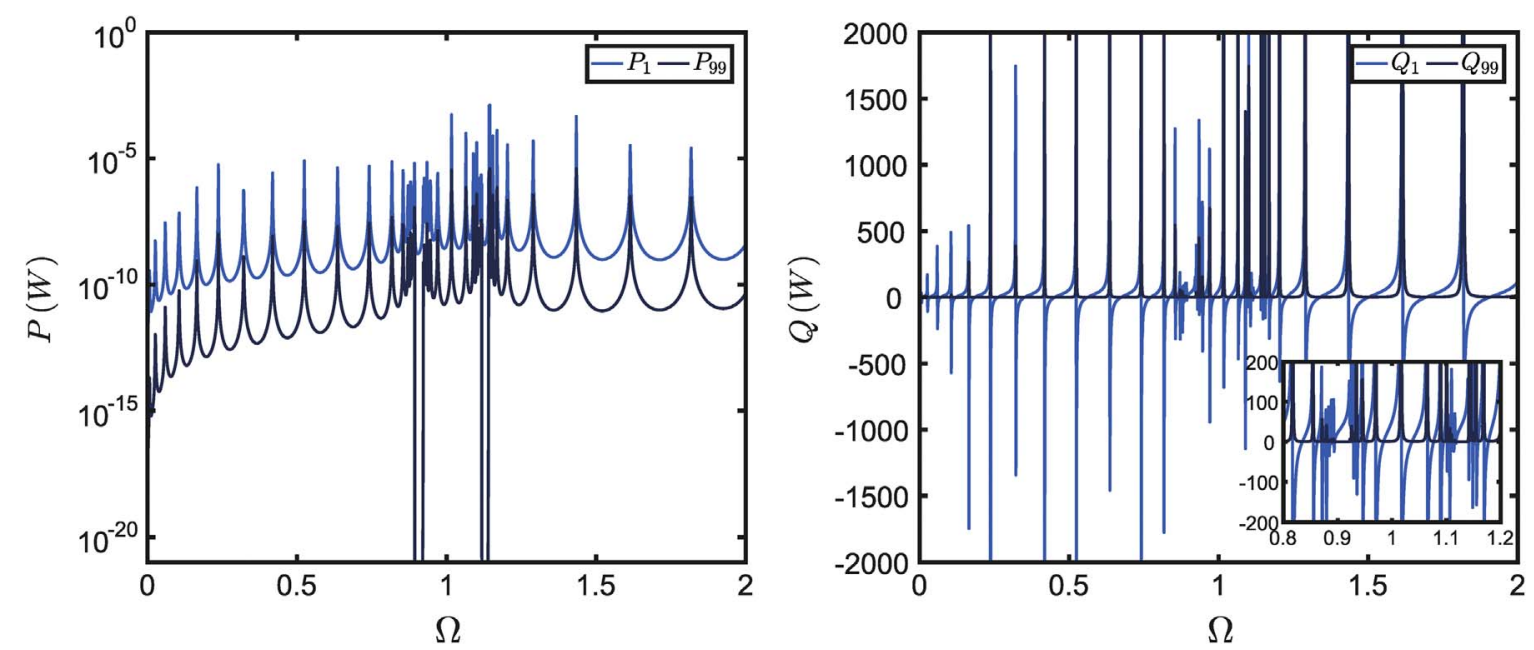

Fig. 20. Active (left) and reactive (right) power flow components at the excited and unexcited ends of the metamaterial beam.
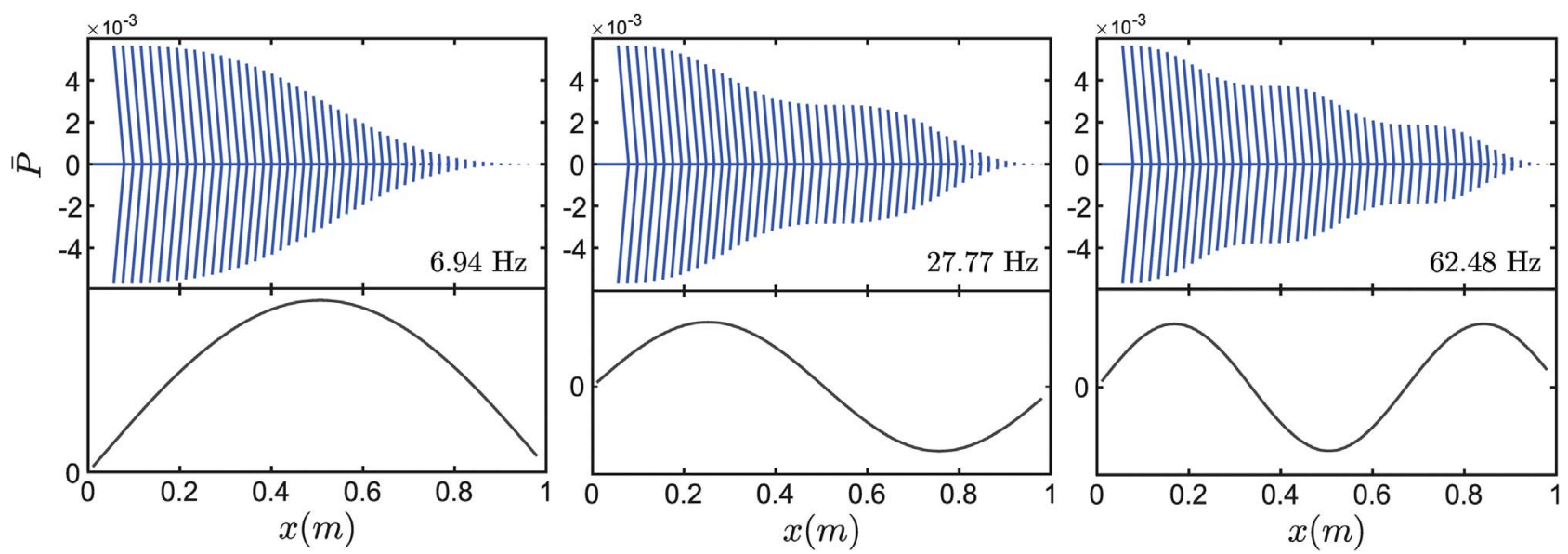

Fig. 21. Power flow maps (top) and corresponding mode shapes (bottom) for the first three bending modes of the uniform beam.

the SIA described in Section 2. The bar is segmented into $n$ number of the cells, each of which has a nodal displacement vector of $\{u\}^{i}=\left\{u_{-1}^{i}, u_{0}^{i}, v^{i}, u_{1}^{i}\right\}^{T}, i=1,2,3 \ldots, n$, as shown in Fig. 3b. The cells has a stiffness matrix of

$K_{\text {cell }}^{i}=\left[\begin{array}{cccc}k_{1} & -k_{1} & 0 & 0 \\ -k_{1} & 2 k_{1}+k & -k & -k_{1} \\ 0 & -k & k & 0 \\ 0 & -k_{1} & 0 & k_{1}\end{array}\right]$

a damping matrix of

$C_{\text {cell }}^{i}=\left[\begin{array}{cccc}0 & 0 & 0 & 0 \\ 0 & c & -c & 0 \\ 0 & -c & c & 0 \\ 0 & 0 & 0 & 0\end{array}\right]$

and a mass matrix that is given by

$M_{\text {cell }}^{i}=\left[\begin{array}{cccc}2 m_{1} / 6 & m_{1} / 6 & 0 & 0 \\ m_{1} / 6 & 4 m_{1} / 6 & 0 & m_{1} / 6 \\ 0 & 0 & m & 0 \\ 0 & m_{1} / 6 & 0 & 2 m_{1} / 6\end{array}\right]$

where $k_{1}=\frac{E A}{L}$. The bar is supported by a spring of a stiffness of $k_{0}=10^{8} E A / \tilde{L}$ which is accounted for in the global stiffness matrix [K].

The metamaterial beam is segmented into $n$ number of the cells, as shown in Fig. 16. Each cell is discretized into two elements with two nodes, and two degrees of freedom for each. We write the mass, stiffness, and damping matrices per cell, which constitute eight degrees of freedom in total. The nodal displacement vector of each $i^{\text {th }}$ cell is presented as $\{u\}^{i}=\left\{w_{-1}^{i}, \theta_{-1}^{i}, w_{0}^{i}, \theta_{0}^{i}, u_{2}^{i}, u_{3}^{i}, w_{1}^{i}, \theta_{1}^{i}\right\}^{T}, i=1,2,3 \ldots, n$. The stiffness, damping matrix, and mass matrices are given by 

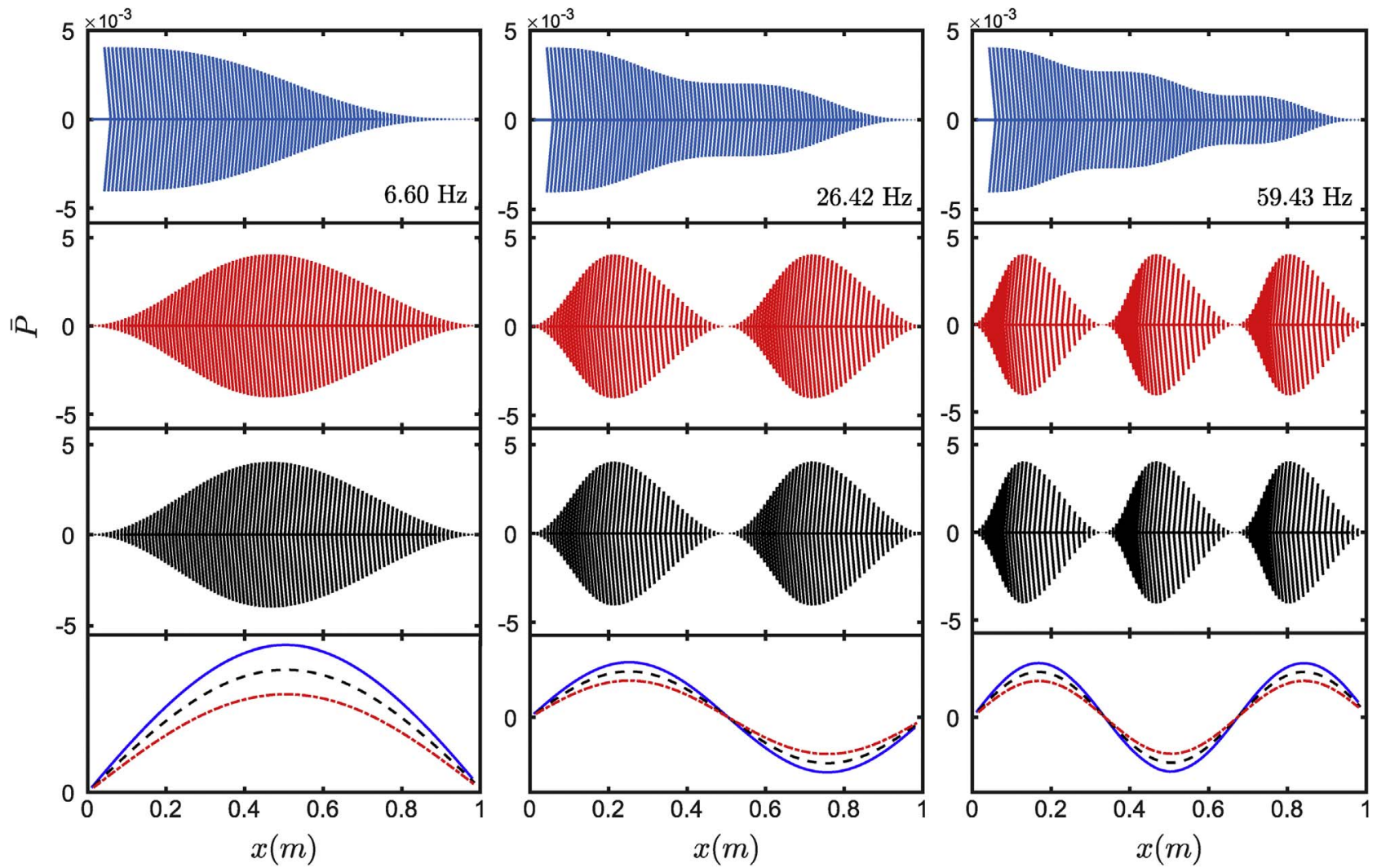

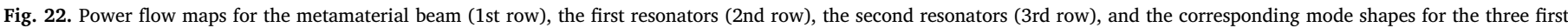
bending modes (last row).
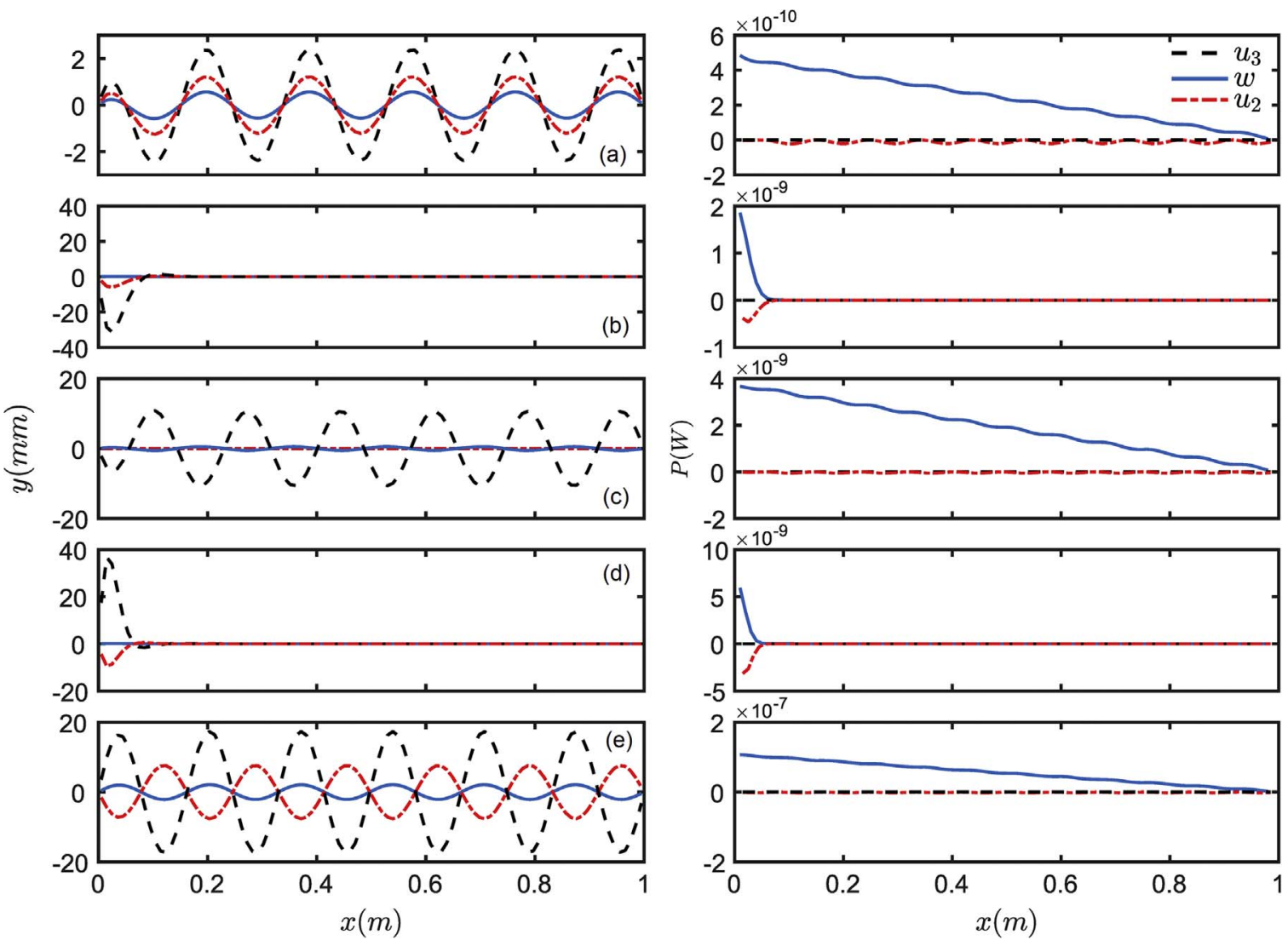

Fig. 23. Steady-state wave profiles (left) and the corresponding active power profiles (right) at $\Omega=$ (a) 0.7 , (b) 0.9 , (c) 1.0 , (d) 1.12 , and (e) 1.3 . 

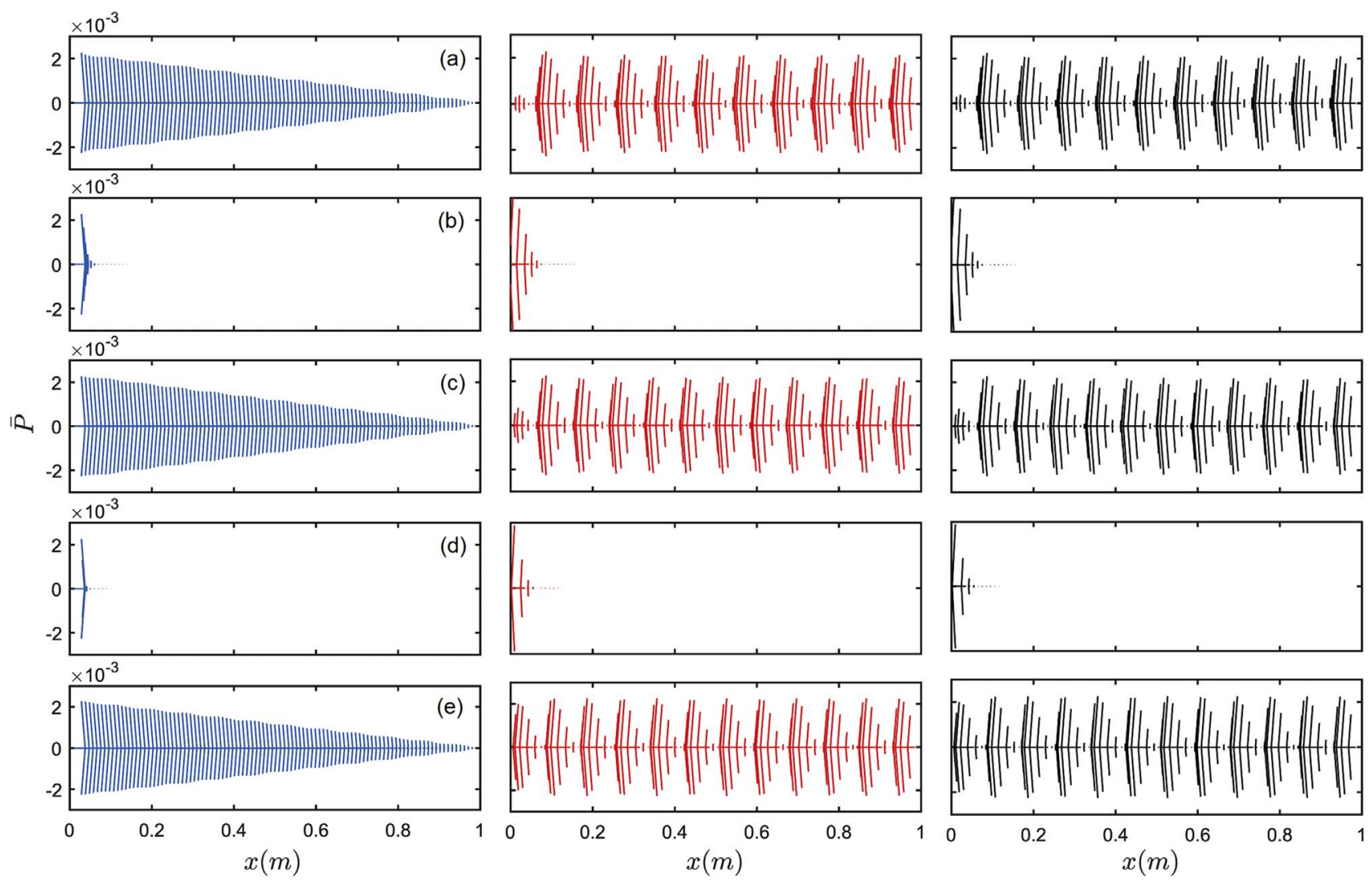

Fig. 24. Power flow maps of the metamaterial beam (left), the first resonator (middle) and the second resonators (right) at $\Omega=$ (a) 0.7 , (b) 0.9 , (c) 1.0 , (d) 1.12 , and (e) 1.3 .

$K_{\text {cell }}^{i}=\kappa\left[\begin{array}{cccccccc}12 & 6 L & -12 & 6 L & 0 & 0 & 0 & 0 \\ 6 L & 4 L^{2} & -6 L & 2 L^{2} & 0 & 0 & 0 & 0 \\ -12 & -6 L & 24+k_{2} / \kappa & 0 & -k_{2} / \kappa & 0 & -12 & 6 L \\ 6 L & 2 L^{2} & 0 & 8 L^{2} & 0 & 0 & -6 L & 2 L^{2} \\ 0 & 0 & -k_{2} / \kappa & 0 & \left(k_{2}+k_{3}\right) / \kappa & -k_{3} / \kappa & 0 & \\ 0 & 0 & 0 & 0 & -k_{3} / \kappa & k_{3} / \kappa & 0 & 0 \\ 0 & 0 & -12 & -6 L & 0 & 0 & 12 & -6 L \\ 0 & 0 & 6 L & 2 L^{2} & 0 & 0 & -6 L & 4 L^{2}\end{array}\right]$

$C_{\text {cell }}^{i}=\left[\begin{array}{cccccccc}0 & 0 & 0 & 0 & 0 & 0 & 0 & 0 \\ 0 & 0 & 0 & 0 & 0 & 0 & 0 & 0 \\ 0 & 0 & c_{2} & 0 & -c_{2} & 0 & 0 & 0 \\ 0 & 0 & 0 & 0 & 0 & 0 & 0 & 0 \\ 0 & 0 & -c_{2} & 0 & \left(c_{2}+c_{3}\right) & -c_{3} & 0 & 0 \\ 0 & 0 & 0 & 0 & -c_{3} & c_{3} & 0 & 0 \\ 0 & 0 & 0 & 0 & 0 & 0 & 0 & 0 \\ 0 & 0 & 0 & 0 & 0 & 0 & 0 & 0\end{array}\right]$

and

$M_{\text {cell }}^{i}=\mu\left[\begin{array}{cccccccc}156 & 22 L & 54 & -13 L & 0 & 0 & 0 & 0 \\ 22 L & 4 L^{2} & 13 L & -3 L^{2} & 0 & 0 & 0 & 0 \\ 54 & 13 L & 312 & 0 & 0 & 0 & 54 & -13 L \\ -13 L & -3 L^{2} & 0 & 8 L^{2} & 0 & 0 & 13 L & -3 L^{2} \\ 0 & 0 & 0 & 0 & m_{2} / \mu & 0 & 0 & 0 \\ 0 & 0 & 0 & 0 & 0 & m_{3} / \mu & 0 & 0 \\ 0 & 0 & 54 & 13 L & 0 & 0 & 156 & -22 L \\ 0 & 0 & -13 L & -3 L^{2} & 0 & 0 & -22 L & 4 L^{2}\end{array}\right]$

where $\kappa$ and $\mu$ are equal to $\frac{\bar{E} I}{L^{3}}$ and $\frac{\rho A L}{420}$, respectively. 


\section{References}

[1] Liu Z, Zhang X, Mao Y, Zhu Y, Yang Z, Chan CT, Sheng P. Locally resonant sonic materials. Science 2000;289(5485):1734-6.

[2] Liu Z, Chan CT, Sheng P. Analytic model of phononic crystals with local resonances. Phys Rev B 2005;71(1):014103.

[3] Huang HH, Sun CT, Huang GL. On the negative effective mass density in acoustic metamaterials. Int J Eng Sci 2009;47(4):610-7.

[4] Huang GL, Sun CT. Band Gaps in a multiresonator acoustic metamaterial. J Vib Acoust 2010;132(3):031003. http://dx.doi.org/10.1115/1.4000784.

[5] Hussein MI, Frazier MJ. Metadamping: an emergent phenomenon in dissipative metamaterials. J Sound Vib 2013;332(20):4767-74.

[6] Huang HH, Sun CT. A study of band-gap phenomena of two locally resonant acoustic metamaterials. Proc Inst Mech Eng N: J Nanoeng Nanosyst 2011. [1740349911409981].

[7] Pai PF. Metamaterial-based broadband elastic wave absorber. J Intell Mater Syst Struct 2010;21(5):517-28. http://dx.doi.org/10.1177/1045389×09359436.

[8] Wang G, Yu D, Wen J, Liu Y, Wen X. One-dimensional phononic crystals with locally resonant structures. Phys Lett A 2004;327(5):512-21.

[9] Xiao Y, Wen J, Wen X. Longitudinal wave band gaps in metamaterial-based elastic rods containing multi-degree-of-freedom resonators. New J Phys 2012;14(3):033042.

[10] Yu D, Liu Y, Zhao H, Wang G, Qiu J. Flexural vibration band gaps in euler-bernoulli beams with locally resonant structures with two degrees of freedom. Phys Rev B 2006;73(6):064301.

[11] Yu D, Liu Y, Wang G, Zhao H, Qiu J. Flexural vibration band gaps in timoshenko beams with locally resonant structures. J Appl Phys 2006;100(12):124901.

[12] Sun H, Du X, and Pai P.F. Theory of metamaterial beams for broadband vibration absorption, J Intell Mater Syst Struct.

[13] Nouh M, Aldraihem O, Baz A. Vibration characteristics of metamaterial beams with periodic local resonances. J Vib Acoust 2014;136(6):61012. http://dx.doi.org/ $10.1115 / 1.4028453$

[14] Nouh M, Aldraihem O, Baz A. Metamaterial structures with periodic local resonances, In: Kundu T, editor. Proc SPIE, vol. 9064; 2014. p. 90641Y. http://dx. doi.org/10.1117/12.2046433.

[15] Pai PF, Peng H, Jiang S. Acoustic metamaterial beams based on multi-frequency vibration absorbers. Int J Mech Sci 2014;79:195-205.

[16] Khajehtourian R, Hussein M. Dispersion characteristics of a nonlinear elastic metamaterial. AIP Adv 2014;4(12):124308.

[17] Xiao Y, Wen J, Yu D, Wen X. Flexural wave propagation in beams with periodically attached vibration absorbers: band-gap behavior and band formation mechanisms. J Sound Vib 2013;332(4):867-93.

[18] Baravelli E, Ruzzene M. Internally resonating lattices for bandgap generation and low-frequency vibration control. J Sound Vib 2013;332(25):6562-79. http:// dx.doi.org/10.1016/j.jsv.2013.08.014.

[19] Zhu R, Liu X, Hu G, Sun C, Huang G. A chiral elastic metamaterial beam for broadband vibration suppression. J Sound Vib 2014;333(10):2759-73. http:// dx.doi.org/10.1016/j.jsv.2014.01.009.

[20] Krushynska A, Kouznetsova V, Geers M. Towards optimal design of locally resonant acoustic metamaterials. J Mech Phys Solids 2014;71:179-96.

[21] Peng H, Pai PF. Acoustic metamaterial plates for elastic wave absorption and structural vibration suppression. Int J Mech Sci 2014;89:350-61.

[22] Wang Y, Wang Y. Complete bandgaps in two-dimensional phononic crystal slabs with resonators. J Appl Phys 2013;114(4):043509.

[23] Nouh M, Aldraihem O, Baz A. Wave propagation in metamaterial plates with periodic local resonances. J Sound Vib 2015;341:53-73. http://dx.doi.org/ 10.1016/j.jsv.2014.12.030.

[24] Hussein MI, Leamy MJ, Ruzzene M. Dynamics of phononic materials and structures: historical origins, recent progress, and future outlook. Appl Mech Rev 2014;66(4):040802. http://dx.doi.org/10.1115/1.4026911.

[25] Gonella S, To AC, Liu WK. Interplay between phononic bandgaps and piezoelectric microstructures for energy harvesting. J Mech Phys Solids 2009;57(3):621-33. http://dx.doi.org/10.1016/j.jmps.2008.11.002.

[26] Chen Y, Huang G, Sun C. Band gap control in an active elastic metamaterial with negative capacitance piezoelectric shunting. J Vib Acoust 2014;136(6):061008.

[27] Celli P, and Gonella S. Tunable directivity in metamaterials with reconfigurable cell symmetry, Appl Phys Lett 106 (9). http://dx.doi.org/10.1063/1.4914011.

[28] Nouh M, Aldraihem O, Baz A. Periodic metamaterial plates with smart tunable local resonators. J Intell Mater Syst Struct 2016;27(13):1829-45.

[29] Mead D. Free wave propagation in periodically supported, infinite beams. J Sound Vib 1970;11(2):181-97. http://dx.doi.org/10.1016/S0022-460X(70)80062-1.

[30] Mead DJ. Vibration response and wave propagation in periodic structures. J Eng Ind 1971;93(3):783. http://dx.doi.org/10.1115/1.3428014.

[31] Faulkner MG, Hong DP. Free vibrations of a mono-coupled periodic system. J Sound Vib 1985;99(1):29-42. http://dx.doi.org/10.1016/0022-460X(85)90443-2.

[32] Bloch F. Über die quantenmechanik der elektronen in kristallgittern. Z Phys 1929;52(7):555-600. http://dx.doi.org/10.1007/BF01339455.

[33] Gavrić L, Pavić G. A finite element method for computation of structural intensity by the normal mode approach. J Sound Vib 1993;164(1):29-43.

[34] Pavic G. The role of damping on energy and power in vibrating systems. J Sound Vib 2005;281(1):45-71.

[35] Pavić G. Structural surface intensity: an alternative approach in vibration analysis and diagnosis. J Sound Vib 1987;115(3):405-22.

[36] Alfredsson KS, Josefson BL, Wilson MA. Use of the energy flow concept in vibration design. AIAA J 1996;34(6):1250-5.

[37] Alfredsson K. Active and reactive structural energy flow. J Vib Acoust 119 1997;1:70-9.

[38] Arruda JR, Dehandschutter W, Sas P. Active vibration control in finite plates using a structural power flow approach. Acta Acust U Acust 1998;84(3):465-74.

[39] Al Babaa H, and Nouh M. An investigation of vibrational power flow in onedimensional dissipative phononic structures, J Vib Acoust.

[40] Li WL, Bonilha MW, Xiao J. Vibrations and power flows in a coupled beam system. J Vib Acoust 2007;129(5):616. http://dx.doi.org/10.1115/1.2775518. 〈http:// vibrationacoustics.asmedigitalcollection.asme.org/article.aspx? articleid $=1470983\rangle$.

[41] Cieślik J, Bochniak W. Vibration energy flow in ribbed plates. Mechanics/AGH University of Science and Technology 25(3); 2006. p. 119-23. 